\title{
Structural Elements and Allosteric Mechanisms Governing Regulation and Catalysis of CSK-Family Kinases and Their Inhibition of Src-Family Kinases
}

Kim K. Ia ${ }^{\ddagger}$, Ryan D. Mills ${ }^{\ddagger}$, Mohammed I. Hossain ${ }^{\ddagger}$, Khai-Chew Chan ${ }^{\ddagger}$, Boonyarin

Jarasrassamee $^{\ddagger}$, Robert N. Jorissen ${ }^{\S}$ and Heung-Chin Cheng ${ }^{\ddagger \dagger}$

From $^{\ddagger}$ Department of Biochemistry \& Molecular Biology, Bio21 Molecular Science and Biotechnology Institute, University of Melbourne, Parkville, Victoria 3010 and ${ }^{\S}$ Ludwig Institute for Cancer Research, Melbourne Tumour Biology Branch, Post Office Box 2008, Royal Melbourne Hospital, Parkville, Victoria 3050, Australia

$\dagger$ Please send all correspondence to:

Dr. Heung-Chin Cheng

Department of Biochemistry and Molecular Biology, Bio21 Institute University of Melbourne

30 Flemington $\mathrm{Rd}$

Parkville, Victoria 3010

Australia

Ph: International: $+(613)-83442254$

Fax: International: +(613)-93481421

E-mail: heung@unuimelb.edu.au

Key words: Protein Kinase, Src-family kinases, CSK, CHK, allosteric regulation, catalysis 


\section{SUMMARY}

C-terminal Src kinase (CSK) and CSK-homologous kinase (CHK) are endogenous inhibitors constraining the activity of the oncogenic Src-family kinases (SFKs) in cells. Both kinases suppress SFKs by selectively phosphorylating their consensus C-terminal regulatory tyrosine. In addition to phosphorylation, CHK can suppress SFKs by a unique non-catalytic inhibitory mechanism that involves tight binding of CHK to SFKs to form stable complexes. In this review, we discuss how allosteric regulators, phosphorylation and inter-domain interactions interplay to govern the activity of CSK and CHK and their ability to inhibit SFKs. In particular, based upon the published results of structural and biochemical analysis of CSK and CHK, we attempt to chart the allosteric networks in CSK and CHK that governing their catalysis and ability to inhibit SFKs. We also discuss how the published three-dimensional structure of CSK complexed with a SFK member sheds light on the structural basis of substrate recognition by protein kinases

(150 words) 


\section{Introduction - CSK and CHK are the major endogenous inhibitors of Src-family kinases}

Normal cell growth, proliferation, differentiation and cell death are governed by an intricate balance of actions of proto-oncogenic and tumour suppressor enzymes. Among the protooncogenic enzymes, the best studied include the Src-family of protein tyrosine kinases. Previous studies of the structure and functions of the Src-family member c-Src and its oncogenic mutant v-Src encoded by the genome of the Rous Sarcoma Virus led to several major discoveries that mark the milestones in cancer research. For example, the $c-S r c$ gene is the first protooncogene identified and studies of the biochemical properties of v-Src protein led to the discovery of protein tyrosine phosphorylation (reviewed in [1-4]). At the biochemical level, the Src-family kinases (referred to as SFKs in this review) are the only notable group of protein tyrosine kinases found to be inhibited by protein tyrosine phosphorylation. The inhibitory tyrosine was mapped to a conserved tyrosine in the C-terminal tail of c-Src [5]. Efforts to study the inhibitory effect of tyrosine phosphorylation on SFKs led to the discovery of C-terminal Src kinase (CSK) by Okada, Nakagawa and their colleagues [6-10]. This was followed by the discovery of CSK-homologous kinase (CHK) by several research groups in 1994 [11-15]. Both CSK and CHK form the CSK-family of protein tyrosine kinases. As their names imply, they suppress activity of SFKs by selectively phosphorylating the conserved C-terminal tail regulatory tyrosine.

The biological and biochemical aspects of regulation and tumour suppressor functions of CSK-family kinases have been covered in several recent reviews [16-19]. The review by Cole and his colleagues in 2003 gave an elegant account of the biochemical and structural aspects of CSK regulation and substrate recognition [20]. In light of the discoveries in the past few years in biochemistry, structures and catalytic mechanism of CSK and CHK, especially the structure of 
CSK complexed with c-Src determined by Levinson et al. [21], it is timely to revisit these topics and discuss the regulation and functions of CSK-family kinases in relation to the recent findings. This manuscript aims to give an overview of the current state of knowledge of the structural basis of substrate recognition of CSK and CHK and their activation by inter-domain interactions and upstream regulators. Based upon the findings from the extensive biochemical and structural studies conducted by us and other researchers, we discuss the functions of the various structural elements essential for regulation and catalysis. We also discuss how the findings enable us to chart the allosteric networks mediating the conformational transition in CSK that govern its activation, catalysis and inhibition of SFKs. It is noteworthy that the CSK/c-Src complex structure reported by Levinson et al. is the first known structure of a protein kinase complexed with its protein substrate [21]. We discuss how the structure sheds light on the molecular basis of substrate recognition and catalysis of protein kinases.

In addition to suppressing SFKs by phosphorylating their C-terminal regulatory tyrosine, CHK is capable of inhibiting SFKs by a non-catalytic mechanism that involves binding of CHK to SFKs to form stable protein complexes $[22,23]$. Deciphering the structural basis of this noncatalytic inhibitory mechanism of $\mathrm{CHK}$ will provide insights into the design of small molecule allosteric inhibitors of SFKs for use as therapeutics for the treatment of cancer. Based upon the published structure of the CSK/c-Src complex [21], we constructed a model of CHK bound to the SFK member Lyn. The model suggests that the mode of intermolecular interaction is likely conserved across both CSK and CHK. In addition, we propose the experimental approaches one can employ to decipher the biochemical and structural basis of the non-catalytic inhibitory mechanism of CHK. 


\section{The conformation and activity of SFKs are governed by phosphorylation at two conserved tyrosines}

SFKs share a common domain arrangement. Beginning from the N-terminus, they contain the fatty acid acylation domain, the unique domain, the SH3 domain, the SH3-SH2 connector, the SH2 domain, the SH2-kinase connector, the kinase domain and the C-terminal tail regulatory domain (Fig. 1A). The kinase domain confers tyrosine kinase activity and contains the activation segment, within which is a consensus autophosphorylation site (e.g. Y416 of chicken Src). Its autophosphorylation leads to auto-activation (reviewed in [24, 25]). The SH2 domain was originally identified as a modular domain in Src but has since been found in many other protein kinases and signaling proteins [26]. SH2 domains contain about 90 amino acids and typically bind to specific peptide motifs that contain phosphorylated tyrosine [27]. The SH3 domain is also a modular domain that mediates protein-protein interactions but contains only about 60 amino acids. The usual binding motifs for SH3 domains are proline-rich sequences with a core consensus sequence of PxxP (where ' $\mathrm{x}$ ' is any amino acid) [28]. The fatty acid acylation domain is a short sequence (about 15 amino acids) at the N-terminus of the protein that contains signals for lipid modifications critical for anchoring the protein to cell membranes [29]. The glycine at position 2 is important for addition of a myristic acid moiety [30] and cysteine residues in this domain are subject to palmitoylation. These cysteines are present in all SFK members except Src and Blk [29]. A short and rather rigid segment called the SH3-SH2 domain connector (referred to as the connector) separates the SH3 and the SH2 domains (Fig. 1). Between the SH2 domain and the kinase domain is the SH2-kinase domain linker region (referred to as the linker), containing an obscure SH3 ligand that participates in intramolecular interactions with the SH3 domain. At the carboxyl terminus of the protein is the C-terminal 
regulatory domain (referred to as the C-terminal tail). It is a short sequence (15-17 amino acids) containing a consensus tyrosine residue (e.g. Tyr-527 of c-Src) that, when phosphorylated, can bind intramolecularly to the SH2 domain and inhibits catalytic activity. Between the fatty acid acylation domain and SH3 domains is the unique domain - a region of variable size (about 4070 amino acids). The N-terminal region containing the fatty acid acylation domain and the unique domain is the most divergent region among them. Apart from lipid modifications (myristoylation/palmitoylation) it also contains structural features unique to the individual SFK members. For example, it contains unique autophosphorylation sites and regulatory serine/threonine phosphorylation sites $[31,32]$ as well as determinants mediating interactions of SFKs with other cellular proteins $[33,34]$. Therefore, the unique domain of SFKs contributes to their regulation, unique subcellular localization and functional specificity.

SFKs are involved in a wide variety of cellular signaling. Their aberrant activation contributes to formation and progression of many types of cancer (reviewed in $[35,36])$. Thus, proper regulation of these protein tyrosine kinases is essential. In normal non-proliferating cells, SFKs are kept in the repressed state with their C-terminal tail tyrosine phosphorylated by CSK and CHK. Fig. 1C depicts the structure of the unphosphorylated form of c-Src [37]. Its Cterminal tail is engaged with the C-terminal lobe of the kinase domain but not the SH2 domain. Its SH2-kinase linker binds to the SH3 domain. Inspection of the configuration of the catalytically critical amino acids revealed that the kinase domain in this structure adopts the active conformation [37]. SFKs can be activated by ligands binding to the SH2 and SH3 domains (Fig. 1C), however, the structures of SFKs with ligands bound to these domains have not been solved. Inactivation of SFKs requires phosphorylation of the C-terminal tail tyrosine by CSK and CHK. In 2008, Levinson et al. solved the crystal structure of CSK/c-Src complex. The 
structure shows that the complex is formed by interactions between unique determinants in the kinase domains of CSK and c-Src (Fig. 1B) [21]. These interactions contribute to selective phosphorylation of the C-terminal tail tyrosine of SFKs by CSK. Upon phosphorylation at the Cterminal tail tyrosine, SFKs adopt the inactive conformation stabilised by two intramolecular inhibitory interactions: (i) binding of the C-terminal tail phosphotyrosine to the SH2 domain (referred to as $\mathrm{pY}_{\mathrm{T}}-\mathrm{SH} 2$ interaction in Fig. 1B), and (ii) binding of the SH2-kinase linker to the SH3 domain (referred to as the linker-SH3 interaction in Fig. 1B) [38, 39]. The structural basis of regulation of SFKs by phosphorylation and inter-domain interactions has been discussed in our recently published review [25].

\section{Features of the active and inactive conformations of CSK and CHK}

Similar to SFKs, CSK and CHK are regulated by phosphorylation and inter-domain interactions. Fig. 2A shows the arrangement of functional domains in CSK and CHK. They both contain $\mathrm{SH} 3, \mathrm{SH} 2$ and kinase domains as well as the intervening segments separating the three domains. Unlike SFKs, they lack the conserved tyrosine phosphorylation site in the activation segment of the kinase domain and the C-terminal tail regulatory tyrosine phosphorylation site. Thus, CHK was referred to as the "hematopoietic consensus tyrosine-lacking kinase" by Sakano et al. [15]. In spite of the similarity in the primary structural arrangements of functional domains in SFKs and CSK-family kinases (Fig. 1A and 2A), the crystal structures of SFKs and CSK revealed significantly different dispositions of the various functional domains in both families of kinases. Thus, it is logical to expect that the structural elements and allosteric mechanisms regulating the kinase activity of SFKs and CSK-family kinases are different. The differences and their implications are discussed in detail in this review. 
Structural elements involved in binding of substrates and catalysis

There are three published reports of crystal structures of CSK [21, 40, 41]. The first report described the crystal structure of the kinase domain of CSK complexed with staurosporine [40]. The second report describes the crystal structures of full-length CSK [41]. The CSK crystals consist of six different structures. Among them, four contain the kinase domain adopting the active conformation and two adopt the inactive conformations [41]. The third report describes the structure of CSK/c-Src complex [21]. Interestingly, the CSK kinase domain in the complex adopted the active conformation with all the structural elements critical for binding ATP and catalysis appropriately aligned for phosphorylation of protein substrates.

Fig. 2B shows the three-dimensional structure of the CSK kinase domain adopting the active conformation $[21,41]$. In this structure, the secondary structures and the structural elements critical for catalysis are appropriately aligned to bind ATP and protein substrates and to catalyse the phosphorylation reaction [42-44]. The functions of these structural elements are discussed below.

For binding of ATP and orienting the $\gamma$-phosphate of ATP for catalysis, the structural elements involved include: (i) the phosphate-binding loop between the $\beta 1$ - and $\beta 2$-strands (glycine-rich loop or P-loop, ${ }^{202} \underline{\mathrm{GKGEFG}}{ }^{207}$ ) which interacts with the $\beta$-phosphate of ATP, (ii) the conserved $\beta 3$-strand lysine (Lys-222) which interacts with the $\alpha$ - and $\beta$-phosphates of ATP, and (iii) the Asp-332 in the DFG motif of the activation segment (Fig. 2B) which binds the magnesium ion chelating the $\beta$ - and $\gamma$-phosphates of ATP. Proper orientation of the $\beta 3$-strand lysine for binding ATP requires its ion pairing with the conserved glutamate (Glu-236) in the $\alpha C$-helix. 
Binding of protein substrates involves the activation segment as well as other regions located distally from the active site pocket. The activation segment begins with the ${ }^{332} \mathrm{DFG}^{334}$ motif and ends with the ${ }^{354} \mathrm{APE}^{356}$-motif (Fig. 2B) [24]. It governs the accessibility of the active site pocket, binding of the magnesium ion interacting with ATP and binding of protein substrates. The activation segment can be subdivided into (i) the DFG-magnesium binding motif, (ii) the $\beta 9$ strand which forms anti-parallel $\beta 9-\beta 6$ strand interactions with the $\beta 6$ strand preceding the catalytic loop in the active conformations of many protein kinases [24], (iii) the activation loop (A-loop), and the $\mathrm{P}+1$ loop responsible for binding to the first residue at the $\mathrm{C}$-terminal side of the target tyrosine residue $\left(\mathrm{P}+1\right.$ residue $\left.^{1}\right)$ in protein substrates. In addition to residues located in and near the active site, several arginine residues in the $\alpha \mathrm{D}$-helix and $\alpha \mathrm{F}-\alpha \mathrm{G}$ loop located distally to the active site pocket of CSK also participates in binding of c-Src (Fig. 2) [21].

Based upon the results of biochemical studies of other protein kinases, it is logical to predict that catalysis of the phosphorylation reaction requires the participation of the conserved Asp-314 in the catalytic loop $\left({ }^{312}\right.$ HRDLAARN $\left.{ }^{319}\right)$ as the catalytic base (Fig. 2)[45]. It accepts the proton from the side-chain hydroxyl group of the phosphorylation site tyrosine to increase its nucleophilicity (reviewed in [45]).

Structural differences between the active and inactive conformations of CSK

The structures of full length CSK in active and inactive conformations revealed that interconversion of CSK between the two conformations involves significant configurational changes of the different motifs within the kinase domain and significant movement of the $\mathrm{SH} 2$ domain

\footnotetext{
${ }^{1}$ The phosphorylation site is referred to as $\mathrm{P}$ site. For the amino acids located at the $\mathrm{N}$-terminal side of the $\mathrm{p}$ site in protein/peptide substrates, they are referred to as P-1, P-2, P-3, etc. For those located at the C-terminal side of the P site, they are referred to as $\mathrm{P}+1, \mathrm{P}+2, \mathrm{P}+3$, etc.
} 
[41]. As shown in Fig. 4A and 4B, the SH2 and kinase domains are in direct contact with each other in the active conformation with residues in the $\beta 3-\alpha \mathrm{C}$ loop of the kinase domain interacting with residues in the Helix-147 and Turn-133 of the SH2 domain (Fig. 4A). However, these contacts are abolished by the upward movement of the SH2 domain when CSK transits from the active to inactive conformations [41]. In addition to changes in inter-domain interactions, the transition of CSK from active to inactive conformations also requires the following structural changes within the kinase domain: (i) disruption of the Lys-222/Glu-236 interaction, (ii) "distortion" of the glycine rich loop, and (iii) significant configurational changes of both the SH3-SH2 domain connector and SH2-kinase domain linker (Fig. 3D, Fig. 2).

A "hydrophobic spine" is formed by four conserved non-contiguous residues in the kinase domain of active CSK

To date, the active and inactive structures of many protein kinases have been solved. These structures have provided a wealth of information on the mechanism of protein kinase activation. Kornev et al. developed a bioinformatics method called "Local Spatial Pattern Alignment" to define the spatial patterns formed by individual residues of the kinase domain in both the active and inactive conformations of many protein kinases $([46,47]$ and reviewed in [48]). With this method, Kornev et al. [46, 47] discovered four conserved residues making hydrophobic interactions to form a 'spine-like' formation that plays an important role in governing protein kinase catalytic activity. This formation, referred to as the hydrophobic spine, comprises of four non-contiguous residues making hydrophobic contacts in the active conformations of many known protein kinases. Assembly of these conserved residues in the "spine-like" formation directs appropriate alignment of the catalytically critical residues for 
catalysis. Once the hydrophobic spine is disassembled or distorted, the catalytically critical residues are misaligned and incapable of binding to substrates and catalysis [46]. Since the assembly and disassembly of this hydrophobic spine is associated with changes in activation state of protein kinases, it is also referred to as regulatory spine by Kornev and Taylor [48].

Close inspection of the structure of the active form of CSK reveals Met-240, Leu-251, Phe-333 and His-312 in the kinase domain as the four conserved residues forming the hydrophobic spine. Viewing from the N-lobe of the CSK kinase domain, the four residues are assembled in the order of (i) Leu-251, (ii) Met-240, (iii) Phe-333 and (iv) His-312 (Fig. 3C). The first residue at the upper end of the spine is Leu- 251 of the $\beta 4$ - strand. It is a component of the R68-W188-L251 network in which Leu-251 is in direct contact with Trp-188 of the SH2-kinase linker and Trp-188 forms cationic- $\pi$ interactions with Arg-68 of the SH3-SH2 connector (Fig. 4A). Leu-251 also forms hydrophobic interaction with Met-241, the next residue of the hydrophobic spine. These interactions allow Leu-251 to act as a conduit that mediates the transmission of allosteric signals from the SH2 and SH3 domains to the kinase domain. Met-241 of $\alpha \mathrm{C}$-helix, the second residue of the spine, is located in $\alpha \mathrm{C}$-helix. It forms hydrophobic interactions with Leu-251 and Phe-333 in the hydrophobic spine. Since $\alpha \mathrm{C}$-helix contains Glu236 that forms the Glu-236/Lys-222 ion pair critical for organising the active site for binding of ATP, proper interactions of Met-240 with Leu-251 and Phe-333 is expected to control the CSK kinase activity by governing the binding of ATP. The third residue of the spine is Phe-333 located in the DFG-motif. As Asp-332 locating next to Phe-333 in the DFG-motif binds the magnesium ion which chelates the $\beta$ - and $\gamma$-phosphates of ATP, proper interactions of Phe-333 with Met-241 and His-312 of the hydrophobic spine are required for the appropriate alignment of $\mathrm{Mg}^{2+}$-ATP for catalysis. The fourth residue of the spine is His-312 located in the catalytic loop 
$\left({ }^{312} \underline{H R}^{314}\right.$ DLAARN) (Fig. 2 and 4). Thus, its interactions with Phe-333 of the hydrophobic spine are expected to affect the alignment of the catalytic base Asp-314 for catalysis. Similar to other protein kinases, the bottom end of the hydrophobic spine of CSK is anchored to the highly hydrophobic $\alpha$ F-helix by a strong hydrogen bond formed by the main chain of His-312 of the spine with the side chain of Asp-368 of the $\alpha$ F-helix (Fig. 3C) [47, 48]. Thus, once properly assembled, the hydrophobic spine appropriately aligns the catalytically critical but noncontiguous residues in the active site for substrate binding and catalysis. Sequence alignment indicates that the hydrophobic spine residues are conserved in both CSK and CHK (SFig. 1).

In many protein kinases, the hydrophobic spine is disassembled in the inactive conformation [46]. However, in the inactive conformations of CSK [41], the spine is not disassembled but only distorted. The distortion involves a translational movement of the side chain of Met-240. Such a distortion is sufficient to induce significant rotational and translational movements of the $\alpha \mathrm{C}$-helix such that the Glu-236/Lys-222 interaction is disrupted (Fig. 3D). Consequently, CSK adopting this conformation is expected to lose the ability to bind and position ATP properly for catalysis.

Since the hydrophobic spine appropriately aligns most of the non-contiguous critical active site residues of protein kinases for ATP/protein substrate binding and catalysis, it has been postulated to form an essential part of the allosteric networks regulating the activity of protein kinases [46]. Thus, mutations of residues that facilitate the assembly of this hydrophobic spine can potentially induce constitutive activation of protein kinases. Relevant to this notion, one of this type of residues is the so-called "gatekeeper" residue which occupies a conserved spatial position in the active conformation of protein kinases. Inspection of all the known structures of protein kinases adopting the active conformation reveals that the gate-keeper residue is in direct 
contact with the N-terminal end of the hydrophobic spine. Thus, substitution of the gate-keeper residue with a bulky and hydrophobic isoleucine residue is expected to stabilise the hydrophobic spine and in turn induce activation of protein kinases. Indeed, replacement of the conserved gatekeeper threonine residue with isoleucine was found to induce a significant increase in kinase activity of several protein tyrosine kinases including c-Src, Abl, PDGF-receptor and FGFreceptor. Thr-266 of CSK and Met-263 of CHK correspond to the gate-keeper residue. It will be interesting to examine if their replacement with isoleucine can induce constitutive activation and enhancement of the tumour suppressor action of CSK and CHK.

Inhibitory structural features constraining the intrinsic catalytic activity of CSK kinase domain Biochemical studies revealed that the isolated kinase domains of many protein kinases exhibit different propensities to adopt the active conformation. Based upon the differences in catalytic activities of the full length enzyme and its corresponding isolated kinase domains, protein kinase domains can be divided into two groups: (i) the intrinsically active (Group 1) and (ii) the intrinsically inactive (Group 2). The group 1 kinase domains contain structural features favouring their adoption of the active conformation (referred to as the activating structural elements) but lack the features restricting them from adopting the active conformation (referred as the inhibitory structural elements). Hence, the Group 1 kinase domains exhibit high intrinsic catalytic activity. The reverse applies to the Group 2 kinase domains exhibiting low intrinsic catalytic activity. The activating and inhibitory structural elements governing the propensity of a kinase domain to adopt the active or the inactive conformations have not been fully defined. In an elegant study performed by Sondhi and Cole, they demonstrated that the isolated CSK kinase domain displayed a catalytic activity $~ 100$-fold lower than that of the full-length CSK [49]. 
Furthermore, interaction of the isolated kinase domain with the $\mathrm{SH} 3$ and $\mathrm{SH} 2$ domains significantly increases its catalytic activity [50-52]. All these lines of evidence indicate that the kinase domain of CSK prefers to adopt the inactive conformation and its intrinsic catalytic activity is enhanced upon its interaction with other functional domains. Results from our previous studies also revealed that the catalytic activity of the isolated CHK kinase domain is much lower than that of full-length CHK (Chong et al. unpublished observations). Thus, both the CSK and CHK kinase domains belong to the Group 1 kinase domains that exhibit low intrinsic catalytic activity.

In contrast to CSK and CHK, the isolated kinase domain of c-Src prefers to adopt an active conformation and exhibits very high kinase activity [23, 53]. Structural and biochemical analyses reveal that CSK and CHK lack an activating structural element found in the N-lobe of the intrinsically active kinase domains of c-Src and several other protein kinases ([54] and reviewed in [25]). In addition, the extraordinarily short activation loop in the activation segment of CSK and CHK constrains their kinase domain to adopt the active conformation (SFig. 3) [21, 24, 55]. When a protein kinase adopts the active conformation and optimally binds the phosphorylation site tyrosine of the substrate, the activation segment needs to interact with the C-lobe of the kinase domain at two anchor points simultaneously. For CSK, the two anchor points are the ${ }^{337} \mathrm{KEA}^{339}$ and the ${ }^{340} \mathrm{SST}^{342}$ motifs in the activation loop (Fig. 2B) [21]. Owing to the extraordinarily short activation loops, the two anchor points of CSK and CHK are likely unable to simultaneously bind to the C-lobe of the kinase domain. Such a structural anomaly was postulated to interfere with protein substrate binding to the active site and this in turn accounts for the low intrinsic catalytic activity of the isolated kinase domains of CSK and CHK [21, 55]. To confirm this notion, Levinson et al. generated CSK mutants with one or more of the residues 
in the variable length loop of the c-Src activation segment inserted in between the two anchor point motifs [21]. They found that the CSK mutants with two or more residues inserted are threefold more active than the wild type CSK, confirming the short activation loop as an inhibitory structural element limiting the intrinsic kinase activity of the CSK kinase domain.

Taken together, the kinase domains of CSK and CHK are intrinsically inactive partly because of (i) the lack of the activating structural element and (ii) the presence of structural anomaly in the activation segment limiting entry of the phosphorylation site tyrosine of the protein substrate into the active site pocket. Since the specific enzymatic activity of full-length CSK is 100-fold higher than that of the isolated CSK kinase domain, it is logical to predict that the presence of the $\mathrm{SH} 2$ and $\mathrm{SH} 3$ domains somehow overcomes these intrinsic structural constraints of the kinase domain to maintain the kinase activity of CSK at a higher level. How the $\mathrm{SH} 2$ and $\mathrm{SH} 3$ domains interact with the kinase domain to overcome these structural constraints is discussed in following sections.

\section{Allosteric Regulation of CSK and CHK}

The roles of SH3 domain and the contiguous segments

The intrinsic catalytic activity of isolated CSK kinase domain is significantly lower than that of full-length CSK [56], indicating that the inter-domain interactions involving SH3 domain, $\mathrm{SH} 2$ domain, the SH3-SH2 connector (referred to as connector) and/or the SH2-kinase linker (referred to as linker) are important in maintenance of the basal activity of CSK at a level higher than that of the isolated kinase domain (Fig. 2). The crystal structure of the active conformation of CSK reveals extensive interactions between the N-lobe of the kinase domain with the other 
functional domains and motifs (Fig. 3A). Furthermore, the adoption of the inactive conformation is associated with alterations in the patterns of the interactions (Fig. 3B).

To more precisely define the role of SH3 domain in regulating CSK kinase activity, Lin et al. generated the CSK mutant with the SH3 domain deleted ( $\triangle \mathrm{SH} 3-\mathrm{CSK})$ [56]. Biochemical analysis revealed that the deletion has no effect on $K_{m}$ but induces a 10 -fold reduction in $k_{\text {cat }}$ in phosphorylation of c-Src, indicating that the SH3 domain does not affect the affinity of the CSK kinase domain for c-Src but it contributes to maintenance of the basal catalytic efficiency of CSK. This notion is further supported by the ability of the isolated segment containing the SH3 domain and the connector to bind and restore the basal activity of the isolated CSK kinase domain [57, 58]. Using surface plasmon resonance, Yaqup et al. demonstrated that the isolated SH3 domain binds the isolated kinase domain with a dissociation constant $\left(K_{D}\right)$ of $8.9 \mu \mathrm{M}$ [58]. Using NMR procedures, Shekhtman et al. identified the $\mathrm{Ser}^{2}-\mathrm{Ala}^{3}$ and $\mathrm{Gly}^{22}-\mathrm{Glu}^{25}$ of the SH3 domain as well as $\mathrm{Gly}^{74}-\mathrm{Met}^{80}$ in the connector as the regions undergoing significant conformational changes upon binding to the isolated CSK kinase domain (SFig.1)[57], indicating that both the SH3 domain and the connector bind to the kinase domain and up-regulate its basal catalytic activity. The crystal structures of full-length CSK show that both the connector and linker sit atop the $\alpha \mathrm{C}$-helix of the kinase domain (Fig. 4A)([41] and reviewed in [17]). It is therefore logical to predict that both the connector and linker participate in the inter-domain interactions between the $\mathrm{SH} 2$ and $\mathrm{SH} 3$ domains with the kinase domain to maintain a high basal kinase activity. Indeed, mutations of Gly-74, Ser-78 or Leu-79 in the linker led to a significant reduction in kinase activity of full-length CSK, confirming its involvement in regulation of CSK kinase activity [57]. 
Fig. 4A and 4B depict how the connector, linker, $\mathrm{SH} 3$ domain and $\mathrm{SH} 2$ domain interact with the kinase domain in the active and inactive conformations of CSK. In the active conformation, two major clusters of residues mediate interactions between the connector and the kinase domain: (i) the $\mathrm{Arg}^{68}-\operatorname{Trp}^{188}-\mathrm{Leu}^{251}$ cluster and (ii) the Leu ${ }^{79}-\mathrm{Phe}^{233}-\mathrm{Leu}^{234}$ cluster. In the first cluster, Arg-68 of the connector forms cation- $\pi$ interactions with Trp-188 of the SH2-kinase linker. Trp-188 also forms hydrophobic interactions with Leu-251 of the hydrophobic spine. Consequently, this cluster forms a conduit conveying conformational changes originating from the SH3 and SH2 domains to the kinase domain of CSK to influence its kinase activity. Indeed, perturbation of the interactions among residues in this cluster by mutations of Arg-68 and Trp188 led to a significant reduction in kinase activity [50]. In the second cluster, Leu-79 of the connector forms hydrophobic interactions with Phe-233 and Leu-234 in the $\alpha \mathrm{C}$-helix. Intuitively, such interactions are expected to influence the electrostatic interaction between Glu-236 in the $\alpha \mathrm{C}$-helix with the $\beta 3$ strand Lys-222. Indeed, mutation of Leu-79 to alanine results in a significant reduction in kinase activity [50], supporting the role of the interactions in the Leu ${ }^{79}$ $\mathrm{Phe}^{233}$-Leu ${ }^{234}$ cluster in regulating the kinase activity of CSK. When CSK adopts the inactive conformation, both the $\mathrm{Arg}^{68}-\mathrm{Trp}^{188}-\mathrm{Leu}^{251}$ cluster and the Leu ${ }^{79}-\mathrm{Phe}^{233}$-Leu ${ }^{234}$ hydrophobic cluster are disrupted (Fig. 4B), further confirming the significance of these two clusters in regulation of CSK kinase activity.

Close inspection of the crystal packing interactions in the CSK crystal structure revealed interactions between the SH3 domains of two neighboring CSK monomeric subunits [21, 59]. Levinson and co-workers noticed that the interactions are mediated in part by the four conserved aromatic hydrophobic residues including Tyr-18, Phe-20, Trp-47 and Tyr-64 involved in binding of the PxxP-motif of the SH3 domain ligands (SFig. 2). Gel filtration column chromatography 
revealed that recombinant CSK forms a dimer in vitro [59]. Deletion of the SH3 domain or mutation of any one of the four conserved aromatic residues abolishes the ability of CSK to form dimers in vitro, confirming that the conserved hydrophobic residues that form the PxxP-motif binding pockets are involved in mediating CSK dimerisation. Additional studies are needed to ascertain if CSK dimerization occurs in vivo and how dimerization affects the kinase activity and cellular functions of CSK.

\section{The role of $\mathrm{SH} 2$ domain}

Deletion of the SH2 domain results in a significant reduction of CSK kinase activity [56], indicating that the $\mathrm{SH} 2$ domain is crucial for stabilising the kinase domain in the active conformation [reviewed in [60]]. Fig. 3 and Fig. 4 show that inactivation of CSK involves significant translocation of the SH2 domain from the kinase domain (referred to as the SH2kinase contact in Fig. 4A). In the active conformation, several residues in the SH2 domain are in direct contact with residues in the N-lobe of the kinase domain. These interacting residues include Ala-228 at the $\beta 3-\alpha \mathrm{C}$ loop of the kinase domain and Tyr-133, Leu-138 and Asn-148 of the SH2 domain. Ala-228 is located in the $\beta 3-\alpha \mathrm{C}$ loop linking the $\alpha \mathrm{C}$-helix and $\beta 3$ strand. The SH2-kinase contact as shown in Fig. 4A is expected to affect the electrostatic interaction between the $\beta 3$ strand Lys-222 and $\alpha \mathrm{C}$-helix Glu-236 which is essential for stabilising the kinase domain in the active conformation. To confirm this notion, Lin et al. performed mutagenesis studies to demonstrate that CSK activity is reduced by mutations of Tyr-133 and Asn-148. Presumably, the mutations either weaken or disrupt the SH2/ $\beta 3-\alpha \mathrm{C}$ loop interactions (Fig. 4A) [50]. When CSK adopts the inactive conformation, the SH2 domain moves away from the kinase domain and disrupts the SH2-kinase contacts (Fig. 4B). 
The subcellular localization and activity of CSK are regulated by its SH2 domain. The $\mathrm{SH} 2$ domain mediates binding of CSK to the transmembrane CSK-binding protein $(\mathrm{Cbp})$ when Tyr-314 of Cbp is phosphorylated by SFKs (reviewed in [16]). The binding recruits CSK to specific compartments of the plasma membrane to inactivate Src-family kinases [61]. Biochemical analysis by Takeuchi et al. demonstrated that binding of phospho-Cbp to CSK-SH2 domain activates CSK kinase activity [62]. Furthermore, binding of a phosphopeptide (AMpYSSV) derived from phospho-Tyr-314 of Cbp to the CSK SH2 domain leads to activation CSK kinase activity [56, 63]. Kinetic analysis reveals that Cbp phosphopeptide binding has little effects on $k_{c a t}$ but decreases the $K_{m}$ of CSK for c-Src [51, 52], suggesting that occupancy of the SH2 domain by the phosphopeptide increases the affinity of CSK for c-Src. To define the regions in the CSK kinase domain that undergo significant changes upon Cbp binding to the $\mathrm{SH} 2$ domain, Wong et al. measured the rates of hydrogen-deuterium exchange in various regions of CSK in the presence and absence of Cbp phosphopeptide [52] (Fig. 4C). From their results, it is possible to chart the allosteric network governing cross-talks between the kinase and SH2 domains. They found that conformational changes in the $\mathrm{SH} 2$ domain (referred to as region 1 in Fig. 4C) induced by the binding of Cbp phosphopeptide could propagate to the $\mathrm{Tyr}^{184}-\mathrm{Asn}^{191}$ segment (region 2) of the SH2-kinase linker and many regions of the kinase domain including (i) the Lys ${ }^{193}-$ Met $^{210}$ segment (region 3) of the glycine-rich loop, (ii) the $\mathrm{Thr}^{241}$-Leu ${ }^{248}$ segment (region 4) near the $\alpha$-helix $\mathrm{C}$ and (iii) the $\mathrm{Tyr}^{403}-\mathrm{Val}^{415}$ segment (region 5) at the $\alpha \mathrm{G}-\alpha \mathrm{H}$ loop (Fig. 4C). It is noteworthy that the $\mathrm{Thr}^{241}-\mathrm{Leu}^{248}$ and $\mathrm{Tyr}^{403}-\mathrm{Val}^{415}$ segments in regions 4 and 5 are distally located from the SH2 domain (Fig. 4C). Presumably, direct contacts between the SH2 and kinase domains as well as the hydrophobic spine together form the passage for the 
transmission of conformational changes from the $\mathrm{SH} 2$ domain to various regions in the kinase domain to modulate its activity (Fig. 4A).

Inspection of the SH2 domain of the crystal structures of all active and inactive conformations of CSK also reveals a peculiar feature - disulfide bond formation between Cys122 and Cys-164. Mills et al. found that the isolated SH2 domain of CSK undergoes conformational changes dependent upon the oxidation state of the $\mathrm{Cys}^{122}-\mathrm{Cys}^{164}$ disulfide bond [63]. More importantly, prevention of reduction of this disulfide bond leads to a ten-fold reduction in kinase activity, which can be restored upon re-introduction of a reducing agent to the reaction mixture. The results indicate that dynamic formation and breakage of this $\mathrm{SH} 2$ domain disulfide bond regulates CSK kinase activity. It is unclear whether Cbp binding alters the dynamics of formation and breakage of the disulfide bond and in turn contributes to the activation of the kinase domain.

Apparently, there is a possibility that dynamic balance of the actions of reactive oxygen species and the anti-oxidants are involved in governing the reversible oxidation and reduction of the $\mathrm{Cys}^{122}$-Cys ${ }^{164}$ disulfide bond. Further investigation is needed to ascertain if CSK regulation by the reversible oxidation-reduction of this disulfide bond is influenced by oxidative stress. It is noteworthy that the ability of CSK to form $\mathrm{Cys}^{122}-\mathrm{Cys}^{167}$ disulfide bond is a late evolutionary phenomenon - Cys-122 and its homologous cysteine residues are only found in the CSK sequences of vertebrates (refer to the sequences of CSK and its orthologs presented in [64, 65]). In the CHK sequence, a phenylalanine occupies the Cys-122 homologous position, suggesting that $\mathrm{CHK}$ activity is not regulated by dynamic disulfide formation and breakage in the $\mathrm{SH} 2$ domain. 
CHK SH2 domain was reported to mediate $\mathrm{CHK}$ binding to specific tyrosine phosphorylation sites of several growth factor receptor kinases including ErbB2, TrkA and c-Kit (reviewed in $[17,19])$. How binding of these autophosphorylated receptors to CHK affects its kinase activity is not known.

It should be pointed out that the document results demonstrating cross-talks between the SH2 and kinase domains were obtained in in vitro studies. Additional studies are needed to confirm their physiological relevance. To this end, it is worthwhile to examine in cells how the mutations preventing the $\mathrm{C} 122-\mathrm{C} 164$ disulfide bond formation and those perturbing the kinaseSH2 domain cross-talks, affect CSK recruitment to the plasma membrane and its phosphorylation and inhibition of the SFKs.

\section{Regulation of CSK by phosphorylation}

CSK is phosphorylated by cAMP-dependent protein kinase (PKA) at Ser-364 at the loop between the $\alpha E F$ and $\alpha F$ helices. The local structure around Ser-364 $\left({ }^{361} \mathrm{KKFS}^{365}\right)$ contains the two critical basic residues commonly found in the consensus PKA phosphorylation site sequence $(++\mathrm{xS} / \mathrm{T} \Phi)^{2}[58]$. Yaqub et al. reported that phosphorylation of Ser-364 resulted in a $\sim 2.5$-fold increase in kinase activity of the isolated kinase domain. However, in the presence of $30 \mu \mathrm{M}$ of the isolated SH3 domain, Ser-364 phosphorylation leads to a $\sim 7.8$-fold increase in catalytic activity of the isolated kinase domain [58]. The results indicate that interactions between the SH3 domain and the kinase domain as depicted in Fig. 4A facilitate the phosphorylated CSK kinase domain to adopt the active conformation. In spite of these findings, the structural basis of activation of CSK by Ser-364 phosphorylation remains unclear. Ser-364 is located near the $\alpha$ F-

\footnotetext{
${ }^{2}$ In this sequence, + represents a lysine or arginine residue; $x$ represents any one of the amino acid residues; and $\Phi$ represents a hydrophobic residue.
} 
helix which forms the anchor of the hydrophobic spine (His-312 of the hydrophobic spine interacts with Asp-368 of the $\alpha$ F-helix) (Fig. 3C) [47]. Presumably, phosphorylation of Ser-364 perturbs the structure of $\alpha \mathrm{F}$-helix and in turn facilitates the assembly of the hydrophobic spine, eventually leading to activation of CSK.

Inspection of the homologous sequences around Ser-364 in CSK from various species reveals that the "KKFST" motif exists in all known CSK sequences from various mammalian species, chicken and zebra fish. However, in the CSK sequences of all other species including those from Drosophila melanogaster, C. elegan and Choanoflagellate species, at least one of the pair of lysine residues in the "KKFST" motif is replaced by a non-basic residue. Thus, CSK proteins from these species are unlikely to be regulated by PKA phosphorylation. In CHK, the KKF $\underline{S} T$ motif is replaced by the "GRF $\underline{S} S$ " sequence in the homologous position (SFig. 1). The GRFSS sequence is not an optimal phosphorylation sequence for PKA. Thus, CHK is unlikely to be regulated by PKA in cells.

\section{Some of the allosteric networks controlling CSK activity are conserved in evolution}

Interactions involved in regulation of CSK activity may be specific to vertebrates only. The clearest example is the disulfide bond between $\mathrm{C} 122$ and C164, which is thought to be involved in catalytic regulation, where formation of the disulphide bond decreases catalytic activity [63]. No cysteine is present at the position equivalent to C122 in CSK homologues from invertebrates. Therefore, catalytic regulation by reduction of the disulphide bond would not occur in these homologues. This leads to consideration of whether the other interactions involved in CSK activation detailed here are widely used throughout the animal kingdom, or unique to vertebrates only. Sequence alignment of vertebrate CSK homologs with those from a range of 
invertebrates shows that residues at the inter-domain interfaces as depicted in Fig. 4A are only partially conserved. The predominant interacting residues of the connector-linker-kinase interface (R68, W188 and L251) are well conserved down to lower organisms (Fig. 4A), with the exception of R68, which is not well conserved in invertebrates, but may be compensated for by a lysine at the equivalent position in the sea squirts Ciona intestinalis and Ciona savignyi and the primitive multicellular sponge Ephydatia fluviatilis. Similarly, for the connector-kinase interface, the hydrophobic nature of L234 and F233 is well conserved across all species examined, however the remaining hydrophobic residue of the interface, L79, is replaced with dissimilar residues in invertebrates. Lastly, while residues important for the SH2-kinase interaction are conserved in vertebrates (Y133, L138, N148, M150 and A228) (Fig. 4A), only the hydrophobic nature of Y133 and L138 are conserved in invertebrates, while residues at the other positions vary widely outside of the vertebrate subphylum. Overall, differences in several of the interdomain interface-forming residues in invertebrates suggest that CSK activity may be regulated differently in these organisms, in comparison with the vertebrate examples already described.

While researchers comparing SFK regulation between unicellular and multicellular organisms have focused on regulation of c-Src itself, some evidence also suggests that the determinants in CSK regulating its kinase activity may have undergone changes during the evolution of multicellular life. Specifically, comparison of kinase activities of recombinant human CSK and a CSK ortholog from the unicellular choanoflagellate Monosiga brevicollis against the artificial substrate poly $\left(\mathrm{Glu}_{4}\right.$-Tyr) suggest that the kinase activity of the $M$. brevicollis ortholog is almost 10-fold lower than that of human CSK [64]. As mentioned above for invertebrates, several of the residues in inter-domain interfaces involved in CSK regulation are not conserved in M. brevicollis CSK, and these differences may explain the considerable 
differences in kinase activity. Further work may determine whether low intrinsic catalytic activity of $M$. brevicollis CSK is unique to unicellular organisms, reflecting the lack of a CSK/SFK regulatory mechanism in these cells, or whether invertebrate CSK possesses similarly low activity, potentially affecting Src regulation across diverse animal phyla. Relevant to this notion, the invertebrate CSK may employ a different mechanism to suppress SFK activity. It is worthwhile to examine if they can employ the non-catalytic inhibitory mechanism similar to that of CHK [22] to down-regulate SFK activity.

\section{Substrate Specificity}

It is well documented that protein kinases recognise both the local structural elements around the phosphorylation site as well as long-range distally located structural elements in protein substrates as substrate specificity determinants $[20,66,67]$. For example, the $\mathrm{p} 130^{\mathrm{Cas}}$ is an efficient protein substrate of SFKs. The local structure around Tyr-668 ( ${ }^{668}$ YDYV) contains the determinant (Val-671) for optimal phosphorylation by SFKs. It also contains the motif $\left({ }^{639} \underline{R} P\right.$ LPSPP) which binds the SH3 domain of SFKs [68]. Pellicena et al. demonstrated that both the local determinants in the ${ }^{668}$ YDYV motif and the distal determinants in the ${ }^{639} \underline{\text { RP LPSPP }}$ cooperate to direct efficient phosphorylation of Tyr-668 of p130 ${ }^{\text {Cas }}$ by SFKs [68]. Although SFKs are the major physiological substrates of CSK and CHK, a growing body of evidence indicates that they can phosphorylate and modulate the activity of other non-SFK cellular proteins $[69,70]$. How the local determinants and distal determinants in SFKs and the non-SFK substrates cooperate to direct CSK and CHK to phosphorylate the target tyrosine residues is the main focus of discussion in this section. 


\section{Contribution of the local structure around the phosphorylation site in protein substrates}

The amino acid sequence of any SFK member contains more than twenty tyrosine residues. Among them, the $\mathrm{C}$-terminal tail tyrosine is the only tyrosine residue recognised exclusively by CSK and CHK as the phosphorylation target. How might CSK and CHK selectively recognise the $\mathrm{C}$-terminal tail tyrosine as the phosphorylation site? To address this question, several groups of researchers used the combinatorial peptide library approach to determine the optimal phosphorylation site sequences of CSK [71-74]. The optimal CSK phosphorylation sequences identified by these studies are EEIYFFF, (-)ФФ $\underline{\Phi \Phi Ф \Phi ~ a n d ~(-)(-~}$ )Ф $\underline{\Phi Ф Ф \Phi ~ w h e r e ~} \Phi$ and (-) represent hydrophobic and acidic residues, respectively. How might CSK recognise the optimal phosphorylation sequence? When the structure of the peptide substrate of a tyrosine kinase [insulin-like growth factor-1 receptor kinase (IGF1RK)] is docked to the active site pocket of CSK, a number of hypothetical interactions between CSK and its peptide substrates are noticeable. As shown in SFig. 4, Lys-347, Leu-348, Pro-349, Val-350 and W352 in the $\mathrm{P}+1$ loop of the activation segment and Leu-392 in the $\alpha \mathrm{F}-\alpha \mathrm{G}$ loop form a hydrophobic cradle capable of binding the target tyrosine and the hydrophobic Ile, and three phenylalanines at the $\mathrm{P}-1, \mathrm{P}+1, \mathrm{P}+2$ and $\mathrm{P}+3$ positions, respectively of the optimal CSK phosphorylation sequence (EEI $\underline{Y F F F) . ~}$

In addition to SFKs, CSK and CHK are known to phosphorylate other cellular proteins. CSK could directly phosphorylate Tyr-26 and Tyr-170 of the transcription factor c-Jun and the phosphorylation regulates ubiquitination and degradation of c-Jun [70]. CSK was recently found to phosphorylate Tyr-393 of the ATP-activated P2X $\mathrm{X}_{3}$ receptor in both the transfected HEK293T cells and mouse trigeminal neurons involved in transduction of painful stimuli [69]. Inspection of the amino acid sequences around the phosphorylation sites reveals uncharged and hydrophobic 
residues at the $\mathrm{P}-1, \mathrm{P}+1$, and $\mathrm{P}+2$ positions of the sequences (Table 1). In addition to the local structures around the phosphorylation site (Tyr-393), D'Arco et al. postulated that the amino acid residues located distally from Tyr-393 of $\mathrm{P} 2 \mathrm{X}_{3}$ receptor also participated in directing its phosphorylation by CSK [69]. Although the authors provided convincing data that CSK is responsible for phosphorylation of $\mathrm{P}_{2} \mathrm{X}_{3}$ receptor at Tyr-393, further biochemical studies are needed to fully establish that $\mathrm{P} 2 \mathrm{X}_{3}$ receptor is directly phosphorylated by CSK in vivo. Likewise, additional studies are needed to confirm that c-Jun is physiological substrate of CSK.

CHK was known to induce phosphorylation of an immunoglobulin superfamily protein SHPS-1. When expressed in PC12 cells, CHK selectively binds to two of the four immunoreceptor tyrosine-based inhibitory motifs (ITIMs) at the cytoplasmic tail of SHPS-1 [75]. The binding is mediated by the CHK SH2 domain. Site-directed mutagenesis studies reveal that CHK phosphorylates Tyr-428, Tyr-452, Tyr-469 and Tyr-495. Again, the amino acid sequences around these phosphorylation sites in SHPS-1 bear little resemblance to the conserved Cterminal tail sequences in SFKs (Table 2). Although the data strongly suggest that CHK directly binds to SHPS-1 and enhances SHPS-1 phosphorylation in transfected cells, additional studies using of RNAi to suppress CHK expression and phosphospecific antibodies to demonstrate SHPS-1 phosphorylation in cells are needed to establish that CHK can directly phosphorylate SHPS-1 at these sites in vivo. In addition to SFKs and SHPS-1, CHK may also phosphorylate other cellular proteins in vivo, the KESTREL method described by Cohen and Knebel will be useful for searching and identification of potential physiological substrates of CHK [76]. Furthermore, defining the CHK optimal phosphorylation sequence by the recently developed positional combinatorial peptide library approach [77] is another avenue to search for additional potential physiological substrates of CHK. 
How do CSK and CHK selectively recognise Src-family kinases as their substrates?

It is noteworthy that the optimal phosphorylation sequence (EEIYFFF) of CSK shares little similarity to the sequences Q $\underline{Y Q P G E}$ and Q $\underline{Y Q Q Q P}$ found in the C-terminal tail of most Src-family kinases. Wang et al. generated a c-Src mutant with the C-terminal tail sequence (EPQYQPGNL) replaced by EEIYFFF [73]. The resultant c-Src mutant and wild type c-Src were phosphorylated by CSK with similar efficiencies, suggesting that the primary structure of the Cterminal tail plays little or no role in directing CSK to selectively phosphorylate the C-terminal tail tyrosine of Src-family kinases. Biochemical and structural studies reveal that determinants located distally from the C-terminal tail tyrosine of SFKs play major roles in directing their binding and phosphorylation by CSK $[21,73,78,79]$. In two insightful and elegant studies, Lee and colleagues predicted three basic residues (Arg-279, Arg-281 and Arg-283 of the $\alpha \mathrm{D}$ helix of CSK) and several residues (Glu-510, Tyr-511 and Gln-518) in the $\alpha$ I-helix of c-Src as the docking residues mediating binding and efficient phosphorylation of c-Src by CSK [78, 79]. Their findings were confirmed by the crystal structure of CSK/c-Src complex solved by Levinson et al. in 2009 [21].

Results of our previous studies demonstrated that CHK phosphorylated the intact Srcfamily kinase Hck much more efficiently than the synthetic peptide derived from the C-terminal tail of SFKs [22], suggesting that the local structure around the C-terminal tail plays only a minor role in the recognition of the C-terminal tail tyrosine by CHK. Given the high degree of sequence homology of CSK and CHK, it is logical to predict that similar to CSK phosphorylation of c-Src, selective and efficient phosphorylation of the C-terminal tail tyrosine 
of c-Src by $\mathrm{CHK}$ is dictated by interactions of residues located distally from the active site pocket of CHK and those located distally from the C-terminal tail tyrosine of c-Src.

The structure of the CSK/c-Src complex is the first structure solved for a protein kinase bound to its protein substrate [21]. It has provided considerable insights into the molecular basis of CSK and CHK recognition of SFKs as the substrates. Furthermore, the structure sheds light on how structural determinants located distally from the phosphorylation site govern protein substrate recognition by protein kinases in general.

The structure of the CSK/c-Src complex exhibits a novel mode of interaction, not seen in other tyrosine kinase structures. One face on the C-terminal lobe of Csk consisting of the $\alpha \mathrm{D}-$ helix and $\alpha \mathrm{F}-\alpha \mathrm{G}$ loop binds to a region toward the C-terminal end of the c-Src kinase domain, including the amino acid residues 504-525 in the $\alpha \mathrm{I}-$ helix of c-Src (Fig. 2 and Fig. 5B). The cSrc residues present in this interface (Fig. 5A and B) are largely conserved across the Src family of kinases (SFig. 5). Moreover, residues in the c-Src-binding motifs in CSK are mostly conserved in CHK. Thus, this interface is likely to be conserved in other SFKs bound to CSK or CHK, as modeled for the complex formed between the SFK member Lyn and CHK (Lyn-CHK complex) shown in Fig. 5D.

A characteristic feature of the c-Src-CSK interface is the presence of five arginine residues in CSK, 279, 281, 283, 384, 389, which are highly conserved in CHK (Arg 317, Arg 319, Arg 321, Arg 422 and Lys 427, respectively). The importance of these residues is underscored by the reduction in binding affinity of CSK to Src upon mutation of any one of these residues to alanine [21]. The interaction of CSK and the c-Src kinase domain varies with salt concentration, suggesting that their affinity depends in part on long-range electrostatic interactions [21]. Four of the interface arginine residues of CSK make short-range interactions 
with acidic residues in c-Src. Arg-281 and Arg-389 make single hydrogen bonds with c-Src residues Glu-504 and Glu-517, respectively. The CSK residues Arg-283 and Arg-384 each make bidentate hydrogen bonds with Glu-510 and Asp-518 of c-Src, respectively. The mammalian members of the Src family of kinases contain acidic residues in these positions, however, the substitution of aspartate for Glu-510 in the corresponding positions of Lck and Lyn (SFig. 5), removes the bidentate hydrogen bonds to Arg-283 of Src (Fig. 5D), and this can potentially affect the binding affinity. The guanidinium side chain of Arg-279 of CSK engages in $\pi-\pi$ stacking with the benzene ring of Tyr-511. An aromatic residue is present in this position in other mammalian SFKs (SFig. 5).

Additional hydrogen bonding interactions occur between the c-Src Lys-442 side chain (conserved in mammalian SFKs) and the carbonyl group of Ser-280, and CSK residue Lys-351 (Lys-449 in CHK) to the carbonyl group of Thr-521 in c-Src. Additionally, the hydrophobic part of the c-Src Thr-521 side chain packs against CSK residues 389-391, particularly its main chain. Similarly the side chain of c-Src Thr 508 packs against residues 280-281 of CSK, but also makes a hydrogen bond with the carbonyl group of Arg-279. These two residues are strongly conserved across the SFKs. Other c-Src surface residues in this region that are conserved are Gln 513, which form hydrogen bonds with Glu-517, and Ala -515, which packs against the guanidinium group of Arg-384 in CSK; this alanine is often substituted by another small residue, serine, in other SFKs. The sequence alignment suggests that the interaction between Pro-525 of c-Src and Leu-392 of CSK will only occur in some members Src, Yes, Fyn, and Fgr of the Src-family.

An alignment of the homologous sequences from other families of tyrosine kinases to the main CSK-interacting region of c-Src shows a lack of sequence conservation of key interacting 
residues, suggesting that the interaction of CSK and CHK to the Src family members will not occur in other tyrosine kinases, not even in the closely related Frk-family kinases.

\section{Determinants mediating CSK and c-Src interactions are highly conserved in evolution}

Evidence exists of other Src-family kinases with regulatory mechanisms that differ between species, ranging from mammalian species to primitive unicellular and multicellular eukaryotes. An ortholog of c-Src exists in the unicellular choanoflagellate Monosiga ovata, along with an ortholog of CSK. These M. ovata orthologs can complement the CSK-Src regulatory system from mice, or the multicellular sponge Ephydatia fluviatilis [65]. Indeed, the basic residues in the $\alpha \mathrm{D}$ helix and $\alpha \mathrm{F}-\alpha \mathrm{G}$ loop of mammalian CSK that recognize the $\alpha \mathrm{I}-\mathrm{helix}$ of c-Src are relatively well-conserved throughout the animal kingdom, including the unicellular choanoflagellates. However, the authors found that although M. ovata CSK could phosphorylate its corresponding Src ortholog; the phosphorylation could only partially inhibit the activity of $M$. ovata c-Src, unlike c-Src orthologs from multicellular organisms. The results indicate that the CSK-Src regulatory system is partially developed in the primitive unicellular eukaryote. The results by Segawa, et al. also suggest that mechanisms of Src regulation occur differently in unicellular organisms compared with multicellular organisms, possibly reflecting the broad functional development of multicellular processes such as cell-to-cell signaling and adhesion. It will be interesting to examine if full development of the CSK-Src regulatory system contributes to the transition from unicellularity to multicellularity in evolution of eukaryotes [80].

The structural basis of the inefficient inhibition of M. ovata c-Src by C-terminal tail phosphorylation is unclear. One possible explanation is that the C-terminal tyrosine phosphorylated M. ovate c-Src cannot adopt the inactive conformation stabilised by 
intramolecular interactions between the SH2 domain and the phosphorylated C-terminal tail tyrosine and those between the $\mathrm{SH} 3$ domain and the linker. Future investigation to address this question may focus on determining the accessibilities of the $\mathrm{SH} 2$ and $\mathrm{SH} 3$ domains to their ligands. The SH2 domain ligand pYEEI peptide and the recently discovered SH3 domain ligand PD1 (HSKYPLPPLPSL) of SFKs are useful tools for analyzing the accessibility of SH2 and SH3 domains of the C-terminal tyrosine-phosphorylated M. ovata c-Src [81, 82].

\section{Catalysis}

Catalysis of CSK has been extensively studied by Cole et al. and Adams et al. Similar to other protein kinases, CSK catalysis of phosphorylation of c-Src requires the participation of the conserved Asp-314 and Arg-318 in the catalytic loop $\left({ }^{312}\right.$ HRDLAARN $)$. Asp-314 forms hydrogen bonds with the phenolic hydroxyl group of the target tyrosine residue of the protein substrate and functions as the catalytic base in the phosphotransfer reaction. Arg-318 forms hydrogen bonds with Asp-314 and the phenolic oxygen of the target tyrosine of the substrate. Studies by Cole and his colleagues demonstrated that the R318A mutation resulted in a substantial reduction in activity ( $\sim 3000$-fold reduction in $\left.k_{c a t}\right)$. Intriguingly, the reduction can be partially restored by the addition of diamino compounds especially imidazole [83]. The results further support the critical role played by the hydrogen bonding network formed by Asp-314, Arg-318 of the catalytic loop and the phenolic oxygen of the target tyrosine in catalysis [reviewed in [84]).

Adams and his colleagues employed the stop-flow approach to study the pre-steady state kinetics of CSK phosphorylation of c-Src $[85,86]$. Their results indicate that efficient binding 
and phosphorylation of c-Src is governed by conformational changes of CSK during the course of the phosphorylation reaction. In this section, we discuss the structural basis of their findings.

Binding of C-terminal tail tyrosine of c-Src to the active site pocket of CSK may induce further structural perturbations affecting catalysis

It is noteworthy that in the structure of CSK/c-Src complex, the C-terminal tail tyrosine (referred to as $\mathrm{Y}_{\mathrm{T}}, \mathrm{Tyr}-527$ ) of c-Src is engaged in intramolecular interactions with Leu-360 and Pro-361 in the $\alpha \mathrm{E}$-helix. Thus, $\mathrm{Y}_{\mathrm{T}}$ needs to translocate from the current location to the active site pocket of CSK for its phosphorylation by CSK (indicated by an arrow in Fig. 6). To predict how $\mathrm{Y}_{\mathrm{T}}$ interacts with residues in the active site pocket of CSK, the structure of CSK active conformation is fitted to the insulin receptor kinase (IRK) complexed with a peptide substrate. Fig. 6 shows the structure of CSK/c-Src complex with the peptide substrate of insulin receptor kinase (IRK) docked to the active site of CSK. From the structure, it is obvious that once bound to the active site pocket, $\mathrm{Y}_{\mathrm{T}}$ can hydrophobically interact with Trp-352 in the $\mathrm{P}+1$ loop of the activation segment of CSK. These interactions may induce further structural perturbation to the active site of CSK and in turn modulate its catalytic efficiency of phosphorylation of $\mathrm{Y}_{\mathrm{T}}$ of c-Src. Relevant to this, Masterson et al. use NMR spectroscopy to map the allosteric networks of structural perturbation of PKA induced by binding of $\mathrm{Mg}^{2+}$-ATP and peptide substrate to the active site [87]. They found that binding of the peptide substrate to the C-lobe induces significant structural perturbation in both the N- and C-lobes of the kinase domain. Since active recombinant ${ }^{2} \mathrm{H} /{ }^{15} \mathrm{~N} /{ }^{13} \mathrm{C}$-labeled CSK kinase domain can be generated in bacterial expression system, a similar approach can be employed to map the regions in CSK undergoing structural perturbation induced by binding of the $\mathrm{C}$-terminal tail to the active site. One can monitor the 
chemical shift changes of recombinant ${ }^{2} \mathrm{H} /{ }^{15} \mathrm{~N} /{ }^{13} \mathrm{C}$-labeled CSK kinase domain induced by binding of the ATP analog (e.g. AMPPNP) and c-Src, [Y527F]c-Src or the c-Src mutant with the C-terminal tail truncated. The differences in patterns of chemical shift changes induced by wild type c-Src and the two c-Src mutants may help investigators to map the regions in CSK that are perturbed by the entry of the c-Src C-terminal tail to the active site pocket of CSK.

In addition to NMR spectroscopy, other biophysical approaches are useful in deciphering the allosteric networks regulating the specificity and efficiency of phosphorylation of Src-family kinases by CSK and CHK. Adams and his colleagues performed pre-steady kinetic and biophysical analyses to understand how CSK recognises and phosphorylates c-Src. Results of their studies demonstrated that CSK catalyses phosphorylation of c-Src rapidly, followed by the quick release of phospho-c-Src from the active site of CSK [85]. Thus, catalysis and product release are not the rate-limiting steps in the c-Src phosphorylation reaction. Using stopped-flow fluorescent spectroscopy to monitor the changes in intrinsic tryptophan fluorescence of c-Src upon binding to CSK and CSK phosphorylation of its C-terminal tyrosine, they studied the kinetics of c-Src interaction with CSK. Their results show that a slow conformational change is associated with the binding of CSK and c-Src. From their data, they postulated a model illustrating the kinetics of binding of CSK and c-Src, and the conformational changes associated with catalysis. In their model, two types of CSK conformations $\{\mathrm{CSK}\}$ and $\left\{\mathrm{CSK}^{*}\right\}$ exist $[85]$. Between them, $\left\{\mathrm{CSK}^{*}\right\}$ is an avid binder of c-Src but catalytically incompetent while $\{\mathrm{CSK}\}$ is a poor binder of c-Src but capable of catalysing phosphorylation of c-Src. At equilibrium, $\{\mathrm{CSK}\}$ is the more predominant species. They postulated that without the phosphorylation reaction, the conformational changes associated with $\left\{\mathrm{CSK}^{*}\right\}$ to $\{\mathrm{CSK}\}$ inter-conversion in the CSK/c-Src complex occur slowly (Fig. 7A). However, when $\{\mathrm{CSK}\}$ is allowed to catalyse 
phosphorylation of c-Src, the inter-conversion takes place rapidly (Fig. 7B). First, the phosphorylation can pull the equilibrium between $\{\mathrm{CSK}\}$ and $\left\{\mathrm{CSK}^{*}\right\}$ in favour of $\{\mathrm{CSK}\}$ in the CSK/c-Src complex. Second, the phosphorylation reaction of c-Src occurring in the active site pocket of $\{\mathrm{CSK}\}$ induces its conversion to $\left\{\mathrm{CSK}^{*}\right\}$. At the time their hypothesis was proposed, the crystal structure of CSK/c-Src complex had not been published. Thus, it is unclear whether the CSK in published structure of CSK/c-Src complex adopt the conformation with properties similar to those of $\left\{\mathrm{CSK}^{*}\right\}$ or $\{\mathrm{CSK}\}$ depicted in the model (Fig. 7).

As discussed in the previous section, CSK is activated by dynamic disulfide bond formation between Cys-122 and Cys-164, the binding of Cbp to the SH2 domain and phosphorylation of Ser-364. It is reasonable to predict that they may activate CSK by enhancing the $\left\{\mathrm{CSK}^{*}\right\}-\{\mathrm{CSK}\}$ inter-conversion when they are bound to c-Src in the catalytic cycle (Fig. 7).

\section{Conclusions, implications and outstanding questions}

Thanks to the recent findings made by several groups of researchers, we now have a clearer picture of the structural basis of selective phosphorylation of the C-terminal tail tyrosine of Src-family kinases by CSK. Being the first known crystal structure of a protein kinase complexed with its protein substrate, the CSK/c-Src complex structure forms the paradigm for future investigation into the molecular basis of substrate selectivity of the protein kinases which exhibit tight interaction with their protein substrates. Furthermore, studies using nuclear magnetic resonance spectroscopy and deuterium exchange approaches have revealed the pathways of transmission of configurational changes by which inter-domain interactions in CSK activates its kinase activity [51, 52]. In spite of these findings, a number of outstanding questions 
still remain to be answered. The most notable one relates to regulation of CSK by PKA - how does PKA phosphorylation of Ser-364 activate CSK? Determination of the crystal structure of CSK stoichiometrically phosphorylated at Ser-364 is an avenue to address this question.

\section{Outstanding questions related to the non-catalytic inhibitory mechanism of CHK}

Previously, we demonstrated that $\mathrm{CHK}$ is capable of employing a non-catalytic inhibitory mechanism to suppress Src-family kinase activity. This mechanism involves binding of CHK to SFKs adopting the active conformation to form stable protein complexes. More importantly, the binding alone is sufficient to down-regulate the Src-family kinase activity $[22,23]$. We have mapped the domain mediating tight binding of Src-family kinases to the kinase domain of CHK [23]. The basic residues in the $\alpha \mathrm{D}$ helix and $\alpha \mathrm{F}-\alpha \mathrm{G}$ loop of CSK critical for mediating binding of CSK to c-Src are conserved in both the CSK and CHK sequences, suggesting that CHK also employs the homologous basic residues in these regions to bind to SFKs. However, the binding of CHK to SFKs is much tighter than that of CSK to SFKs $[22,23]$. It is logical to predict that in addition to the determinants in the $\alpha \mathrm{D}$ helix and $\alpha \mathrm{F}-\alpha \mathrm{G}$ loop, $\mathrm{CHK}$ enlists determinants in other regions of its kinase domain to further enhance its binding to SFKs. Thus, further studies are needed to map these determinants. Whether the determinants critical for binding are also involved in inhibition is another outstanding question. Further biochemical investigations are needed to define the role of the basic residues in the $\alpha \mathrm{D}$ helix and $\alpha \mathrm{F}-\alpha \mathrm{G}$ loop of CHK in binding and suppression of the activity of SFKs. Finally, determination of the three-dimensional structures of $\mathrm{CHK}$ and $\mathrm{CHK} / \mathrm{c}-\mathrm{Src}$ complex will provide valuable insights into the non-catalytic inhibitory mechanism of CHK. 
What are the non-Src kinase substrates of CHK?

In addition to phosphorylating SFKs, CHK is known to phosphorylate other cellular proteins and perform additional cellular functions. The most notable one is induction of neurite outgrowth in PC12 cells and activation of the MAP kinase signaling pathway [75, 88]. Furthermore, CHK induced activation of MAP kinase is independent of Src-family kinases as this occurs even in the SFY cells in which Src-family kinases are not expressed [88]. One mechanism by which CHK induces MAP kinase activation in these cells is by phosphorylation of one of more unknown cellular proteins governing activation of the MAP kinase signaling pathway. Relevant to this, CHK was found to bind and induce tyrosine phosphorylation of a cellular protein called tyrosine-protein phosphatase non-receptor type substrate 1 (SHPS-1) in PC12 cells. The phosphorylation sites have been identified and shown in Table 2. Furthermore, the phosphorylation of SHPS-1 by CHK is associated with the induction of neurite outgrowth of the PC12 cells [75]. It is unclear how CHK recognises SHPS-1 and other non-SFK protein substrates. If these non-SFK protein substrates are recruited to CHK by interactions with the $\mathrm{SH} 2$ and/or SH3 domains or motifs distal to the active site in the kinase domain, individual domains of CHK can be used as "baits" to isolate these CHK protein substrates. On the other hand, if these non-SFK protein substrates contain determinants specifically recognised by the CHK active site, defining the CHK optimal phosphorylation sequence will facilitate the search for these non-SFK protein substrates - cellular proteins containing the CHK optimal phosphorylation sequence are potential physiological substrates of CHK. Since there are only a few known structures of tyrosine kinases complexed with peptide substrates, the available protein kinase substrate prediction programs such as Predikin are unable to predict the optimal 
phosphorylation sequence of CHK $[89,90]$. Future investigation using the newly developed positional combinatorial peptide library approach [77] will help defining the CHK optimal phosphorylation sequence.

Implications for the design of selective inhibitors of SFKs as therapeutics

A number of small molecule inhibitors targeting the active site (especially the ATPbinding pocket of the active site) of SFKs have been developed and used in preclinical studies. Among them, Dasatinib, AZD0530 and SKI-606 are under clinical trials for use as treatment of different forms of cancer [35]. The use of Dasatinib for the treatment of patients with drugresistant chronic myelogenous leukaemia has been approved (reviewed in [35]). The major drawback of the use of these inhibitors for therapeutics to combat against cancer relates to the specificity of these inhibitors. In addition to efficiently inhibiting SFKs, these inhibitors can also inhibit the activity of many other protein kinases and non-kinase cellular proteins [91]. The abilities of these inhibitors to bind and perturb the activity and function of other protein kinases and non-kinase proteins account for the side effects when they are used as therapeutics for the treatment of cancer patients. To circumvent this problem, researchers aim at developing the allosteric inhibitors of protein kinases for therapeutic uses. These inhibitors allosterically suppress protein kinase activity and function by binding to regions outside the ATP-binding pocket. Since most if not all these regions are unique to individual kinases, inhibitors targeting these regions are likely highly specific (reviewed in [92]). Close inspection of the structure of cSrc in the CSK/c-Src complex revealed that c-Src adopts a unique inactive conformation [21, 25]. As this conformation is induced by binding of CSK to the $\alpha$ I-helix of c-Src, it is logical to predict that the region consisting of the $\alpha$ I-helix and its surrounding motifs can be exploited to 
develop the allosteric inhibitors of SFKs. Small molecules binding to this region and inducing the SFK to adopt the unique inactive conformation similar to that found in the CSK/c-Src complex are allosteric inhibitors of SFKs. Thus, future studies to investigate the substrate recognition of CSK and $\mathrm{CHK}$ and the non-catalytic inhibitory mechanism of CHK will benefit the design of allosteric SFKs inhibitors for therapeutic uses. 


\section{Acknowledgements}

The authors' work described in this review was supported by research grants from the National Health and Medical Research Council of Australia and Cancer Council Victoria. RM is a recipient of the Dowd Neuroscience Postgraduate Research Fellowship. 


\section{Figure Legends}

\section{Figure 1 Mechanism of inactivation of SFKs by CSK and CHK}

A. Organisation of functional domains of SFKs.

All SFK members have similar arrangement of their functional domains and regulatory elements. Starting from the N-terminus, SFKs contain a fatty acid acylation motif to which the myristoyl and palmitoyl groups are attached. This is followed by the unique domain which exhibits the greatest structural diversity among the SFK members. In several SFK members including Hck, Lyn, Fgr and Fyn, the unique domain contains a autophosphorylation site ( $\left.\mathrm{Y}_{\mathrm{U}}\right)$. SFKs are highly homologous in all other domains. The SH3 domain is linked to the C-terminal end of the unique domain. The SH3-SH2 connector motif (Connector) links the SH3 to the SH2 domain. The SH2-kinase domain linker (Linker) links the SH2 domain to the kinase domain. The kinase domain contains a consensus autophosphorylation site $\left(\mathrm{Y}_{\mathrm{A}}\right)$ in the activation segment. The C-terminal regulatory domain (referred to as C-terminal tail) contain the consensus C-terminal tail tyrosine $\left(\mathrm{Y}_{\mathrm{T}}\right)$ which is phosphorylated by CSK and CHK.

B. Inactivation of SFKs by CSK and CHK

The unphosphorylated form of SFKs is inhibited by CSK and CHK by a two-step mechanism. The first step involves the formation of a complex of SFKs with CSK/CHK. Our previous study discovered that the complexes between SFKs with CHK are much tighter and SFKs in the complex are inactive. In second step, CSK and CHK selectively phosphorylate the C-terminal tail tyrosine of SFKs. The phosphorylated SFKs then dissociate from CSK and CHK and adopt the inactive conformation. The structural basis of how the phosphorylated SFKs dissociate from $\mathrm{CSK} / \mathrm{CHK}$ to adopt the inactive conformation is unknown. The inactive 
conformation of SFKs is stabilised by two intramolecular inhibitory interactions: (i) the $\mathrm{pY}_{\mathrm{T}^{-}}$ $\mathrm{SH} 2$ interaction of which the phosphorylated C-terminal tail tyrosine binds to the SH2 domain, and (ii) the linker:SH3 interaction of which the $\mathrm{SH} 2$-kinase domain linker adopts a polyproline type II helical structure and then binds to the SH3 domain. These images were prepared by the magic fit function of Swiss pdb viewer [93] and Molsoft program (http://www.molsoft.com/index.html).

C. Mechanisms of SFK activation and their inactivation by CSK and CHK

SFKs are activated by multiple mechanisms including binding of the ligands to the $\mathrm{SH} 2$ and SH3 domains to displace the two inhibitory intramolecular interactions, autophosphorylation and dephosphorylation of the C-terminal tail tyrosine $\left(\mathrm{Y}_{\mathrm{T}}\right)$. CSK and CHK employs two mechanisms inhibit the active forms of SFKs: (i) Selective phosphorylation of $\mathrm{Y}_{\mathrm{T}}$ by CSK and CHK, and (ii) the non-catalytic inhibitory mechanism of CHK. This mechanism involves the binding of CHK to the active forms of SFKs to form the inactive SFK/CHK complexes. The binding alone is sufficient for inhibition.

Figure 2 Organisation of functional domains of CSK-family kinases and the structure and functional motifs of the CSK kinase domain
A. Organisation of functional domains of CSK and CHK
B. Amino acid sequence of the CSK kinase domain (residues 192-445). The $\beta$-strands and $\alpha$ - helices making up the kinase domain are indicated below the corresponding segments. The structural elements critical for catalysis, regulation and binding of substrates are listed above the corresponding segments. These elements include: (i) the phosphate- binding loop (P-loop) of the consensus motif ( $\mathrm{GxGx} \Phi \mathrm{G}$, where $\mathrm{x}$ is any amino acid 
residue and $\Phi$ represents a hydrophobic residue) responsible for interacting with the $\beta$ phosphate of ATP; (ii) the $\beta 3$ strand Lys (Lys-222) which, upon formation of an ion pair with (iii) the $\alpha \mathrm{C}$-helix glutamate (Glu-236), interacts with the $\alpha$ - and $\beta$-phosphates of ATP; (iv) the "hinge" motif that links the N-terminal lobe (residues 192-267) to the Cterminal lobe (274-445) of the kinase domain; (v) the catalytic loop (HRLAARN) containing the conserved aspartate that functions as the general base in catalysis; (vi) the activation segment beginning with the DFG motif and ending with the APE motif; and (vii) the arginine residues (Arg-279, Arg-281, Arg-283, Arg-284, Arg-289) in the $\alpha \mathrm{D}-$ helix and $\alpha \mathrm{F}-\alpha \mathrm{G}$ loop critical for binding to c-Src (indicated by green arrow heads).

C. Structure of the active CSK kinase domain (in the CSK/c-Src complex, PDB entry:3D7T). All $\beta$-strands and $\alpha$-helices are labeled. The $\alpha \mathrm{D}$-helix and $\alpha \mathrm{F}-\alpha \mathrm{G}$ loop making up the c-Src-binding motifs are indicated. The hinge region links the $\mathrm{N}$-terminal lobe (N-lobe) and the $\mathrm{C}$-terminal lobe (C-lobe) of the kinase domain together. The crevice between the two lobes forms the active site.

\section{Figure 3. The active and inactive conformation of CSK}

A. The active conformation of CSK. The black arrow indicates the hydrophobic spine formed by four conserved residues. The red arrow indicates the direction of movement of the SH2 domain

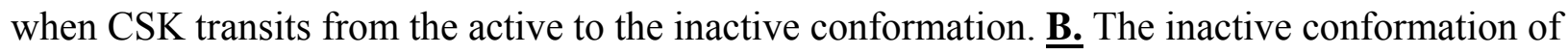
CSK. The "eye" indicates the direction at which the close-up views of the kinase domain were

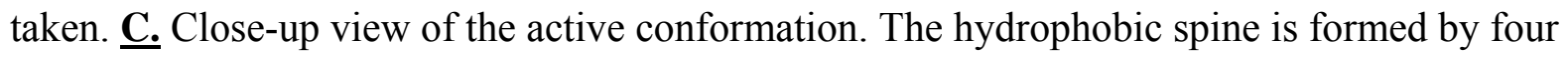
conserved residues including Leu-251 of the $\beta 4$ sheet, Met-240 of the ahelix-C, Phe-333 of the DFG-motif in the activation segment, and His-312 of the catalytic loop. The contact between 
His-321 and Asp-386 forms the line of communication between the hydrophobic spine and the conserved $\alpha$ F-helix. Similar to all active conformation of the protein kinases, the side chains of $\beta 3$ strand Lys-222 and $\alpha \mathrm{C}$-helix Glu-236 form an ion-pair critical for binding of ATP. The glycine residues in the glycine-rich loop located between $\beta 1$ and $\beta 2$ are shown. D. A close-up view of the inactive conformation of CSK. The Lys-222 and Glu-236 ion pair is disrupted due to movement of $\beta 3$ sheet and $\alpha$ helix-C. The glycine-rich loop, the linker and connector undergo significant movement when CSK undergoes inter-conversion between the active and inactive conformation (dotted arrows).

\section{Figure 4 Cross-talk between the SH2 and kinase domains of CSK}

A. The close-up diagram showing the intramolecular contacts among the SH3, SH2 and kinase domains in the active conformation of CSK. These contacts are divided into four groups (presenting clock-wise from the top left hand corner): (i) C122-C164 disulfide bond in the SH2 domain, (ii) SH2-kinase contacts, (iii) connector-linker-kinase tripartite contacts and (iv) connector-kinase contacts. The C122-C164 disulfide bond is believed to undergo breakage and formation which may contribute to inter-conversion of CSK between the active and inactive conformations. The SH2-kinase contacts exist only in the active conformation. The contact points in the SH2 domain include Asn-148 and Met-150 of the $\alpha$-helix beginning from residue 147 (Helix-147), and Tyr-133 and Leu-138 of the $\beta$-turn beginning from residue 133 (Turn-133). The contact points (Ala-228 and its neighboring residues) in the kinase domain reside in the $\beta 3$ $\alpha \mathrm{C}$ loop linking the $\beta 3$ sheet and $\alpha$ helix-C. The connector-linker-kinase tripartite contacts consist of Arg-68 of the connector, Trp-188 of the linker and Leu-251 of the hydrophobic spine. 
The side chains of Arg-68 and Trp-188 form the cationic- $\pi$ interaction. These contacts can form a conduit of communication between the SH3 domain and the kinase domain. The connectorkinase contacts are formed by Leu-79 of the connector and Phe- 233 of $\alpha$-helixC. The contacts could affect the formation of the ion pair by Lys-222 and Glu-236 in the active conformation. B. The close-up diagram showing inter-domain contacts in the inactive conformation. In addition to disruption of the Lys-222/Glu-236 ion pair, the SH2-kinase contacts, connectorlinker-kinase contacts and the connector-kinase contacts are all perturbed or disrupted in the inactive conformation. C122-C164 disulfide bond in the SH2 domain, binding of $\mathrm{L} 79$ of the SH3-SH2 connector to a hydrophobic pocket formed by F233 and A234 of the $\alpha$-helix C and the $\pi$-cationic interaction between R68 and W188. Since W188 is making hydrophobic contacts with L251 of the hydrophobic spine and both F233 and A234 are contiguous with M240 of the hydrophobic spine, structural perturbations induced by ligand binding to the $\mathrm{SH} 3$ and $\mathrm{SH} 2$ domains could use these contacts with the hydrophobic spine to transmit the signals to other regions in the kinase domain.

C. Conformational changes in CSK induced by binding of the phosphopeptide derived from Cbp to the SH2 domain. The conformational changes were revealed by Cbp phosphopeptideinduced modulation of the hydrogen/deuterium exchange rates at different regions of recombinant CSK. These regions are assigned with numbers. They include: region 1: the N111S121 and S139-E143 segments in the SH2 domain; region 2: the Y184-N191 segment of the SH2-kinase linker; region 3: the K193-M210 segment corresponding to the glycine-rich loop; region 4: the T241-L248 segment near the $\alpha$-helix C; and region 5: the Y403-V415 segment at the $\alpha \mathrm{G}-\alpha \mathrm{H}$ loop. The colors of the motifs indicate how Cbp binding changes the dynamics of the 
conformational changes: blue indicates an increase in dynamics of H/D exchange while red indicates reduction in the dynamics of $\mathrm{H} / \mathrm{D}$ exchange.

Figure 5 Structural basis of interactions between CSK/CHK with Src-family kinases Interacting residues in the complex formed by the kinase domains of CSK (green) and c-Src (cobalt blue) are shown. A. Left panel, the CSK/c-Src complex. Right panel, $\mathrm{C}_{\alpha}$ trace (tube representation) of CSK and c-Src kinase domains. B. Detail of interacting regions of c-Src (the $\mathrm{C}_{\alpha}$ trace is shown only for residues 503-526) and CSK. Selected hydrogen bonds are shown as yellow dashes. C. Alignment of C-terminal tail sequences of c-Src and Lyn, the sequences corresponding to $\alpha \mathrm{D}$ helix and $\alpha \mathrm{F}-\alpha \mathrm{G}$ loop of CSK and CHK. The acid and basic residues involved in the formation of the electrostatic interactions at the c-Src/CSK interface of the complex and the Lyn/CHK interface in the putative Lyn/CHK complex are underlined. D. Superimposition of a modeled CHK (pink): Lyn (tan) complex onto the CSK:c-Src complex with (putative) interacting residues shown. This model was generated using the MODELLER comparative modeling program [94]. The molecular representations shown in this figure were generated using PyMOL [95].

Figure 6 Structure of CSK-c-Src complex with the IRK substrate peptide docked to the CSK active site pocket

The figure shows a portion of the c-Src/CSK interface at which configurational changes in c-Src could be transmitted to CSK. The insulin receptor kinase (IRK) peptide substrate is docked to the active site of CSK. Trp-352 (W252) is a conserved residue located in the $\mathrm{P}+1$ loop of the activation segment of CSK. It is postulated that binding of the target tyrosine of the protein 
substrate to the active site is expected to perturb $\mathrm{P}+1$ loop. W252 is located in a hydrophobic pocket formed by L315, A316, W370 and P386 that are linked to the hydrophobic spine residues (H312 and F333). The changes can be transmitted through the hydrophobic spine to other regions in CSK. In addition, since P386 of the hydrophobic pocket is contiguous to R384 and R389 which are critical c-Src binding residues, configurational changes in c-Src can be transmitted from the R384 and R389, via the hydrophobic pocket and the hydrophobic spine to other regions of CSK. Ser-364 of the $\alpha$-helixF is phosphorylated by PKA. Its phosphorylation contributes to CSK activation.

\section{Fig. 7 A model depicting the mechanism of activation and catalysis of CSK.}

In this model, CSK molecules exist in two major conformational states, $\left\{\mathrm{CSK}^{*}\right\}$ and $\{\mathrm{CSK}\}$ which are in equilibrium. For the CSK molecules adopting the $\left\{\mathrm{CSK}^{*}\right\}$ state, the Srcbinding site is open while its active site pocket is occluded. Thus, $\left\{\mathrm{CSK}^{*}\right\}$ can bind c-Src $\alpha \mathrm{I}-$ helix with high affinity it exhibits very low catalytic activity. For the CSK molecules adopting the $\{\mathrm{CSK}\}$ state, the Src-binding site is deformed and closed while the active site pocket is open. Thus, it can catalyse phosphorylation of c-Src. However, its affinity for the $\alpha$ I-helix of c-Src is low. A. Binding and distribution of $\left\{\mathrm{CSK}^{*}\right\}$ and $\{\mathrm{CSK}\}$ states without catalysis. Without catalysis, the equilibrium favours the $\{\mathrm{CSK}\}$ conformation. Thus, the apparent affinity of the

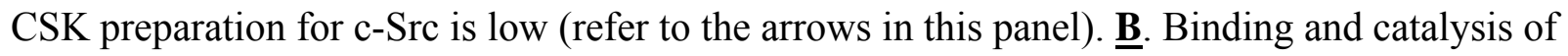
CSK to c-Src. First, the two conformations of CSK are in equilibrium (Step 1). Both $\left\{\mathrm{CSK}^{*}\right\}$ and $\{\mathrm{CSK}\}$ bind c-Src with different affinities (Step 2). As $\left\{\mathrm{CSK}^{*}\right\}$ is catalytically incompetent, the c-Src/ $\left\{\mathrm{CSK}^{*}\right\}$ complex needs to convert to $\mathrm{c}-\mathrm{Src} /\{\mathrm{CSK}\}$ for catalysis of phosphorylation of C-terminal tail tyrosine of c-Src (Step 3). Biochemical analysis revealed that the catalysis step is 
fast. Even though $\{\mathrm{CSK}\}$ cannot bind $\alpha \mathrm{I}-\mathrm{helix}$ of c-Src with high affinity, c-Src remains bound to $\{\mathrm{CSK}\}$ because of the new interactions formed among the C-terminal tail of c-Src, ATP and CSK active site pocket (Fig. 6). The phosphorylation is accompanied by the conversion of CSK from the $\{\mathrm{CSK}\}$ state to the $\left\{\mathrm{CSK}^{*}\right\}$ state (Step 4). Biophysical studies revealed that phosphorylated c-Src is rapidly released from the active site pocket of CSK (step 5). The arrows in this panel indicate that the fast steps 3,4 and 5 alters the equilibrium of $\left\{\mathrm{CSK}^{*}\right\}$ versus $\{\mathrm{CSK}\}$ and that of c-Src/ $\left\{\mathrm{CSK}^{*}\right\}$ versus c-Src/ $\{\mathrm{CSK}\}$ to favour the formation of c-Src/ $\{\mathrm{CSK}\}$ for catalysis. Consequently, the apparent affinity of CSK for c-Src is significantly increased. 


\section{REFERENCES}

[1] G.S. Martin, The hunting of the Src, Nat Rev Mol Cell Biol 2 (2001) 467-475.

[2] G.S. Martin, The road to Src, Oncogene 23 (2004) 7910-7917.

[3] T.J. Yeatman, A renaissance for SRC, Nat Rev Cancer 4 (2004) 470-480.

[4] T. Hunter, B.M. Sefton, Transforming gene product of Rous sarcoma virus phosphorylates tyrosine, Proc Natl Acad Sci U S A 77 (1980) 1311-1315.

[5] J.A. Cooper, K.L. Gould, C.A. Cartwright, T. Hunter, Tyr527 is phosphorylated in pp60c-src: implications for regulation, Science 231 (1986) 1431-1434.

[6] S. Nada, M. Okada, A. MacAuley, J.A. Cooper, H. Nakagawa, Cloning of a complementary DNA for a protein-tyrosine kinase that specifically phosphorylates a negative regulatory site of p60c-src, Nature 351 (1991) 69-72.

[7] M. Okada, S. Nada, Y. Yamanashi, T. Yamamoto, H. Nakagawa, CSK: a proteintyrosine kinase involved in regulation of src family kinases, J Biol Chem 266 (1991) 24249-24252.

[8] M. Okada, H. Nakagawa, Protein tyrosine kinase in rat brain: neonatal rat brain expresses two types of pp60c-src and a novel protein tyrosine kinase, J Biochem 104 (1988) 297-305.

[9] M. Okada, H. Nakagawa, Identification of a novel protein tyrosine kinase that phosphorylates pp60c-src and regulates its activity in neonatal rat brain, Biochem Biophys Res Commun 154 (1988) 796-802.

[10] M. Okada, H. Nakagawa, A protein tyrosine kinase involved in regulation of pp60csrc function, J Biol Chem 264 (1989) 20886-20893.

[11] B.D. Bennett, S. Cowley, S. Jiang, R. London, B. Deng, J. Grabarek, J.E. Groopman, D.V. Goeddel, H. Avraham, Identification and characterization of a novel tyrosine kinase from megakaryocytes, J Biol Chem 269 (1994) 1068-1074.

[12] S. Klages, D. Adam, K. Class, J. Fargnoli, J.B. Bolen, R.C. Penhallow, Ctk: a protein-tyrosine kinase related to Csk that defines an enzyme family, Proc Natl Acad Sci U S A 91 (1994) 2597-2601.

[13] S.S. Kuo, P. Moran, J. Gripp, M. Armanini, H.S. Phillips, A. Goddard, I.W. Caras, Identification and characterization of Batk, a predominantly brain-specific nonreceptor protein tyrosine kinase related to Csk, J Neurosci Res 38 (1994) 705-715.

[14] D.W. McVicar, B.K. Lal, A. Lloyd, M. Kawamura, Y.Q. Chen, X. Zhang, J.E. Staples, J.R. Ortaldo, J.J. O' Shea, Molecular cloning of Isk, a carboxyl-terminal src kinase (csk) related gene, expressed in leukocytes, Oncogene 9 (1994) 2037-2044.

[15] S. Sakano, A. Iwama, J. Inazawa, T. Ariyama, M. Ohno, T. Suda, Molecular cloning of a novel non-receptor tyrosine kinase, HYL (hematopoietic consensus tyrosinelacking kinase), Oncogene 9 (1994) 1155-1161.

[16] E. Ingley, Src family kinases: regulation of their activities, levels and identification of new pathways, Biochim Biophys Acta 1784 (2008) 56-65.

[17] Y.P. Chong, T.D. Mulhern, H.C. Cheng, C-terminal Src kinase (CSK) and CSKhomologous kinase (CHK)--endogenous negative regulators of Src-family protein kinases, Growth Factors 23 (2005) 233-244. 
[18] Y.P. Chong, K.K. Ia, T.D. Mulhern, H.C. Cheng, Endogenous and synthetic inhibitors of the Src-family protein tyrosine kinases, Biochim Biophys Acta 1754 (2005) 210-220.

[19] H.-C. Cheng, Y.P. Chong, K.K. Ia, O.a. Tan, T.D. Mulhern, Csk homologous kinase, UCSD-Nature Molecule Pages doi:10.1038/mp.a000705.01 (2006).

[20] P.A. Cole, K. Shen, Y. Qiao, D. Wang, Protein tyrosine kinases Src and Csk: a tail's tale, Curr Opin Chem Biol 7 (2003) 580-585.

[21] N.M. Levinson, M.A. Seeliger, P.A. Cole, J. Kuriyan, Structural basis for the recognition of c-Src by its inactivator Csk, Cell 134 (2008) 124-134.

[22] Y.P. Chong, T.D. Mulhern, H.J. Zhu, D.J. Fujita, J.D. Bjorge, J.P. Tantiongco, N. Sotirellis, D.S. Lio, G. Scholz, H.C. Cheng, A novel non-catalytic mechanism employed by the C-terminal Src-homologous kinase to inhibit Src-family kinase activity, J Biol Chem 279 (2004) 20752-20766.

[23] Y.P. Chong, A.S. Chan, K.C. Chan, N.A. Williamson, E.C. Lerner, T.E. Smithgall, J.D. Bjorge, D.J. Fujita, A.W. Purcell, G. Scholz, T.D. Mulhern, H.C. Cheng, Cterminal Src kinase-homologous kinase (CHK), a unique inhibitor inactivating multiple active conformations of Src family tyrosine kinases, J Biol Chem 281 (2006) 32988-32999.

[24] B. Nolen, S. Taylor, G. Ghosh, Regulation of protein kinases; controlling activity through activation segment conformation, Mol Cell 15 (2004) 661-675.

[25] H.C. Cheng, T.M. Johnson, R.D. Mills, Y.P. Chong, K.C. Chan, J.G. Culvenor, Allosteric Networks Governing Regulation and Catalysis of Src-Family Protein Tyrosine Kinases - Implications for Disease-Associated Kinases, Clin Exp Pharmacol Physiol (2009).

[26] I. Sadowski, J.C. Stone, T. Pawson, A noncatalytic domain conserved among cytoplasmic protein-tyrosine kinases modifies the kinase function and transforming activity of Fujinami sarcoma virus P130gag-fps, Mol Cell Biol 6 (1986) 4396-4408.

[27] M.F. Moran, C.A. Koch, D. Anderson, C. Ellis, L. England, G.S. Martin, T. Pawson, Src homology region 2 domains direct protein-protein interactions in signal transduction, Proc Natl Acad Sci U S A 87 (1990) 8622-8626.

[28] R. Ren, B.J. Mayer, P. Cicchetti, D. Baltimore, Identification of a ten-amino acid proline-rich SH3 binding site, Science 259 (1993) 1157-1161.

[29] M.D. Resh, Interaction of tyrosine kinase oncoproteins with cellular membranes, Biochim Biophys Acta 1155 (1993) 307-322.

[30] M.P. Kamps, J.E. Buss, B.M. Sefton, Mutation of NH2-terminal glycine of p60src prevents both myristoylation and morphological transformation, Proc Natl Acad Sci U S A 82 (1985) 4625-4628.

[31] S. Shenoy, I. Chackalaparampil, S. Bagrodia, P.H. Lin, D. Shalloway, Role of p34cdc2-mediated phosphorylations in two-step activation of pp60c-src during mitosis, Proc Natl Acad Sci U S A 89 (1992) 7237-7241.

[32] T.M. Johnson, N.A. Williamson, G. Scholz, A. Jaworowski, R.E. Wettenhall, A.R. Dunn, H.C. Cheng, Modulation of the catalytic activity of the Src family tyrosine kinase Hck by autophosphorylation at a novel site in the unique domain, $\mathrm{J}$ Biol Chem 275 (2000) 33353-33364.

[33] J.R. Gingrich, K.A. Pelkey, S.R. Fam, Y. Huang, R.S. Petralia, R.J. Wenthold, M.W. Salter, Unique domain anchoring of Src to synaptic NMDA receptors via the 
mitochondrial protein NADH dehydrogenase subunit 2, Proc Natl Acad Sci U S A 101 (2004) 6237-6242.

[34] K.S. Campbell, A. Buder, U. Deuschle, Interactions between the amino-terminal domain of p56lck and cytoplasmic domains of CD4 and CD8 alpha in yeast, Eur J Immunol 25 (1995) 2408-2412.

[35] L.C. Kim, L. Song, E.B. Haura, Src kinases as therapeutic targets for cancer, Nat Rev Clin Oncol 6 (2009) 587-595.

[36] S.J. Parsons, J.T. Parsons, Src family kinases, key regulators of signal transduction, Oncogene 23 (2004) 7906-7909.

[37] S.W. Cowan-Jacob, G. Fendrich, P.W. Manley, W. Jahnke, D. Fabbro, J. Liebetanz, T. Meyer, The crystal structure of a c-Src complex in an active conformation suggests possible steps in c-Src activation, Structure 13 (2005) 861-871.

[38] F. Sicheri, I. Moarefi, J. Kuriyan, Crystal structure of the Src family tyrosine kinase Hck, Nature 385 (1997) 602-609.

[39] W. Xu, S.C. Harrison, M.J. Eck, Three-dimensional structure of the tyrosine kinase c-Src, Nature 385 (1997) 595-602.

[40] M.B. Lamers, A.A. Antson, R.E. Hubbard, R.K. Scott, D.H. Williams, Structure of the protein tyrosine kinase domain of C-terminal Src kinase (CSK) in complex with staurosporine, J Mol Biol 285 (1999) 713-725.

[41] A. Ogawa, Y. Takayama, H. Sakai, K.T. Chong, S. Takeuchi, A. Nakagawa, S. Nada, M. Okada, T. Tsukihara, Structure of the carboxyl-terminal Src kinase, Csk, J Biol Chem 277 (2002) 14351-14354.

[42] S.S. Taylor, D.R. Knighton, J. Zheng, L.F. Ten Eyck, J.M. Sowadski, Structural framework for the protein kinase family, Annu Rev Cell Biol 8 (1992) 429-462.

[43] L.N. Johnson, Protein kinase inhibitors: contributions from structure to clinical compounds, Q Rev Biophys 42 (2009) 1-40.

[44] S.W. Cowan-Jacob, Structural biology of protein tyrosine kinases, Cell Mol Life Sci 63 (2006) 2608-2625.

[45] J.A. Adams, Kinetic and catalytic mechanisms of protein kinases, Chem Rev 101 (2001) 2271-2290.

[46] A.P. Kornev, N.M. Haste, S.S. Taylor, L.F. Eyck, Surface comparison of active and inactive protein kinases identifies a conserved activation mechanism, Proc Natl Acad Sci U S A 103 (2006) 17783-17788.

[47] A.P. Kornev, S.S. Taylor, L.F. Ten Eyck, A helix scaffold for the assembly of active protein kinases, Proc Natl Acad Sci U S A 105 (2008) 14377-14382.

[48] A.P. Kornev, S.S. Taylor, Defining the conserved internal architecture of a protein kinase, Biochim Biophys Acta 1804 (2010) 440-444.

[49] D. Sondhi, P.A. Cole, Domain interactions in protein tyrosine kinase Csk, Biochemistry 38 (1999) 11147-11155.

[50] X. Lin, Y. Wang, Y. Ahmadibeni, K. Parang, G. Sun, Structural basis for domaindomain communication in a protein tyrosine kinase, the C-terminal Src kinase, $J$ Mol Biol 357 (2006) 1263-1273.

[51] L. Wong, S. Lieser, B. Chie-Leon, O. Miyashita, B. Aubol, J. Shaffer, J.N. Onuchic, P.A. Jennings, V.L. Woods, Jr., J.A. Adams, Dynamic coupling between the SH2 domain and active site of the COOH terminal Src kinase, Csk, J Mol Biol 341 (2004) 93-106. 
[52] L. Wong, S.A. Lieser, O. Miyashita, M. Miller, K. Tasken, J.N. Onuchic, J.A. Adams, V.L. Woods, Jr., P.A. Jennings, Coupled motions in the SH2 and kinase domains of Csk control Src phosphorylation, J Mol Biol 351 (2005) 131-143.

[53] A. Weijland, G. Neubauer, S.A. Courtneidge, M. Mann, R.K. Wierenga, G. SupertiFurga, The purification and characterization of the catalytic domain of Src expressed in Schizosaccharomyces pombe. Comparison of unphosphorylated and tyrosine phosphorylated species, Eur J Biochem 240 (1996) 756-764.

[54] K. Huang, Y.H. Wang, A. Brown, G. Sun, Identification of N-terminal lobe motifs that determine the kinase activity of the catalytic domains and regulatory strategies of Src and Csk protein tyrosine kinases, J Mol Biol 386 (2009) 1066-1077.

[55] X. Lin, S. Lee, G. Sun, Functions of the activation loop in Csk protein-tyrosine kinase, J Biol Chem 278 (2003) 24072-24077.

[56] X. Lin, M.K. Ayrapetov, S. Lee, K. Parang, G. Sun, Probing the communication between the regulatory and catalytic domains of a protein tyrosine kinase, Csk, Biochemistry 44 (2005) 1561-1567.

[57] A. Shekhtman, R. Ghose, D. Wang, P.A. Cole, D. Cowburn, Novel mechanism of regulation of the non-receptor protein tyrosine kinase Csk: insights from NMR mapping studies and site-directed mutagenesis, J Mol Biol 314 (2001) 129-138.

[58] S. Yaqub, H. Abrahamsen, B. Zimmerman, N. Kholod, K.M. Torgersen, T. Mustelin, F.W. Herberg, K. Tasken, T. Vang, Activation of C-terminal Src kinase (Csk) by phosphorylation at serine-364 depends on the Csk-Src homology 3 domain, Biochem J 372 (2003) 271-278.

[59] N.M. Levinson, P.R. Visperas, J. Kuriyan, The tyrosine kinase Csk dimerizes through Its SH3 domain, PLoS One 4 (2009) e7683.

[60] P. Filippakopoulos, S. Muller, S. Knapp, SH2 domains: modulators of nonreceptor tyrosine kinase activity, Curr Opin Struct Biol 19 (2009) 643-649.

[61] M. Kawabuchi, Y. Satomi, T. Takao, Y. Shimonishi, S. Nada, K. Nagai, A. Tarakhovsky, M. Okada, Transmembrane phosphoprotein Cbp regulates the activities of Src-family tyrosine kinases, Nature 404 (2000) 999-1003.

[62] S. Takeuchi, Y. Takayama, A. Ogawa, K. Tamura, M. Okada, Transmembrane phosphoprotein $\mathrm{Cbp}$ positively regulates the activity of the carboxyl-terminal Src kinase, Csk, J Biol Chem 275 (2000) 29183-29186.

[63] J.E. Mills, P.C. Whitford, J. Shaffer, J.N. Onuchic, J.A. Adams, P.A. Jennings, A novel disulfide bond in the SH2 Domain of the C-terminal Src kinase controls catalytic activity, J Mol Biol 365 (2007) 1460-1468.

[64] W. Li, S.L. Young, N. King, W.T. Miller, Signaling properties of a non-metazoan Src kinase and the evolutionary history of Src negative regulation, J Biol Chem 283 (2008) 15491-15501.

[65] Y. Segawa, H. Suga, N. Iwabe, C. Oneyama, T. Akagi, T. Miyata, M. Okada, Functional development of Src tyrosine kinases during evolution from a unicellular ancestor to multicellular animals, Proc Natl Acad Sci U S A 103 (2006) 12021-12026.

[66] R.I. Brinkworth, R.A. Breinl, B. Kobe, Structural basis and prediction of substrate specificity in protein serine/threonine kinases, Proc Natl Acad Sci U S A 100 (2003) 74-79. 
[67] B. Kobe, T. Kampmann, J.K. Forwood, P. Listwan, R.I. Brinkworth, Substrate specificity of protein kinases and computational prediction of substrates, Biochim Biophys Acta 1754 (2005) 200-209.

[68] P. Pellicena, W.T. Miller, Processive phosphorylation of p130Cas by Src depends on SH3-polyproline interactions, J Biol Chem 276 (2001) 28190-28196.

[69] M. D'Arco, R. Giniatullin, V. Leone, P. Carloni, N. Birsa, A. Nair, A. Nistri, E. Fabbretti, The C-terminal Src inhibitory kinase (Csk)-mediated tyrosine phosphorylation is a novel molecular mechanism to limit $\mathbf{P} 2 \mathrm{X} 3$ receptor function in mouse sensory neurons, J Biol Chem 284 (2009) 21393-21401.

[70] F. Zhu, B.Y. Choi, W.Y. Ma, Z. Zhao, Y. Zhang, Y.Y. Cho, H.S. Choi, A. Imamoto, A.M. Bode, Z. Dong, COOH-terminal Src kinase-mediated c-Jun phosphorylation promotes c-Jun degradation and inhibits cell transformation, Cancer Res 66 (2006) 5729-5736.

[71] D. Sondhi, W. Xu, Z. Songyang, M.J. Eck, P.A. Cole, Peptide and protein phosphorylation by protein tyrosine kinase Csk: insights into specificity and mechanism, Biochemistry 37 (1998) 165-172.

[72] Z. Songyang, S.E. Shoelson, J. McGlade, P. Olivier, T. Pawson, X.R. Bustelo, M. Barbacid, H. Sabe, H. Hanafusa, T. Yi, et al., Specific motifs recognized by the SH2 domains of Csk, 3BP2, fps/fes, GRB-2, HCP, SHC, Syk, and Vav, Mol Cell Biol 14 (1994) 2777-2785.

[73] D. Wang, X.Y. Huang, P.A. Cole, Molecular determinants for Csk-catalyzed tyrosine phosphorylation of the Src tail, Biochemistry 40 (2001) 2004-2010.

[74] M. Ruzzene, Z. Songyang, O. Marin, A. Donella-Deana, A.M. Brunati, B. Guerra, P. Agostinis, L.C. Cantley, L.A. Pinna, Sequence specificity of C-terminal Src kinase (CSK)--a comparison with Src-related kinases c-Fgr and Lyn, Eur J Biochem 246 (1997) 433-439.

[75] H. Mitsuhashi, E. Futai, N. Sasagawa, Y. Hayashi, I. Nishino, S. Ishiura, Cskhomologous kinase interacts with SHPS-1 and enhances neurite outgrowth of PC12 cells, J Neurochem 105 (2008) 101-112.

[76] P. Cohen, A. Knebel, KESTREL: a powerful method for identifying the physiological substrates of protein kinases, Biochem J 393 (2006) 1-6.

[77] B.E. Turk, J.E. Hutti, L.C. Cantley, Determining protein kinase substrate specificity by parallel solution-phase assay of large numbers of peptide substrates, Nat Protoc 1 (2006) 375-379.

[78] S. Lee, M.K. Ayrapetov, D.J. Kemble, K. Parang, G. Sun, Docking-based substrate recognition by the catalytic domain of a protein tyrosine kinase, C-terminal Src kinase (Csk), J Biol Chem 281 (2006) 8183-8189.

[79] S. Lee, X. Lin, N.H. Nam, K. Parang, G. Sun, Determination of the substratedocking site of protein tyrosine kinase C-terminal Src kinase, Proc Natl Acad Sci U S A 100 (2003) 14707-14712.

[80] D. Pincus, I. Letunic, P. Bork, W.A. Lim, Evolution of the phospho-tyrosine signaling machinery in premetazoan lineages, Proc Natl Acad Sci U S A 105 (2008) 9680-9684.

[81] H. Schmidt, S. Hoffmann, T. Tran, M. Stoldt, T. Stangler, K. Wiesehan, D. Willbold, Solution structure of a Hck SH3 domain ligand complex reveals novel interaction modes, J Mol Biol 365 (2007) 1517-1532. 
[82] N. Sotirellis, T.M. Johnson, M.L. Hibbs, I.J. Stanley, E. Stanley, A.R. Dunn, H.C. Cheng, Autophosphorylation induces autoactivation and a decrease in the Src homology 2 domain accessibility of the Lyn protein kinase, J Biol Chem 270 (1995) 29773-29780.

[83] D.M. Williams, D. Wang, P.A. Cole, Chemical rescue of a mutant protein-tyrosine kinase, J Biol Chem 275 (2000) 38127-38130.

[84] M.K. Tarrant, P.A. Cole, The chemical biology of protein phosphorylation, Annu Rev Biochem 78 (2009) 797-825.

[85] S.A. Lieser, J. Shaffer, J.A. Adams, SRC tail phosphorylation is limited by structural changes in the regulatory tyrosine kinase Csk, J Biol Chem 281 (2006) 38004-38012.

[86] S.A. Lieser, C. Shindler, B.E. Aubol, S. Lee, G. Sun, J.A. Adams, Phosphoryl transfer step in the C-terminal Src kinase controls Src recognition, J Biol Chem 280 (2005) 7769-7776.

[87] L.R. Masterson, A. Mascioni, N.J. Traaseth, S.S. Taylor, G. Veglia, Allosteric cooperativity in protein kinase A, Proc Natl Acad Sci U S A 105 (2008) 506-511.

[88] R. Zagozdzon, R. Kaminski, Y. Fu, W. Fu, C. Bougeret, H.K. Avraham, Csk homologous kinase (CHK), unlike Csk, enhances MAPK activation via Rasmediated signaling in a Src-independent manner, Cell Signal 18 (2006) 871-881.

[89] N.F. Saunders, R.I. Brinkworth, T. Huber, B.E. Kemp, B. Kobe, Predikin and PredikinDB: a computational framework for the prediction of protein kinase peptide specificity and an associated database of phosphorylation sites, BMC Bioinformatics 9 (2008) 245.

[90] N.F. Saunders, B. Kobe, The Predikin webserver: improved prediction of protein kinase peptide specificity using structural information, Nucleic Acids Res 36 (2008) W286-290.

[91] O. Hantschel, U. Rix, G. Superti-Furga, Target spectrum of the BCR-ABL inhibitors imatinib, nilotinib and dasatinib, Leuk Lymphoma 49 (2008) 615-619.

[92] J. Zhang, P.L. Yang, N.S. Gray, Targeting cancer with small molecule kinase inhibitors, Nat Rev Cancer 9 (2009) 28-39.

[93] W. Kaplan, T.G. Littlejohn, Swiss-PDB Viewer (Deep View), Brief Bioinform 2 (2001) 195-197.

[94] A. Sali, T.L. Blundell, Comparative protein modelling by satisfaction of spatial restraints, J Mol Biol 234 (1993) 779-815.

[95] W.L. DeLano, The PyMOL Molecular Graphics System, http://www.pymol.org (2002). 


\begin{tabular}{|r|r|l|}
\hline Substrate & Sequence & \multicolumn{1}{|c|}{ Comments } \\
\hline c-Src (Y527) & PEERPTFEYLQAFLEDYFTSTEPQY $Q P G N L$ & with distal docking sites \\
P2X $_{3}$ Receptor (Y393) & ASTNPVFASDQATVEKQSTDSG-AYYSIGH & with distal docking sites \\
c-Jun (Y170) & GGFSASLHSEPPVY 1 ANLSN & with distal docking sites? \\
c-Jun (Y26) & LNASFLPSESGPYGYSN & with distal docking sites? \\
Peptide library 2 & EEIY FFF & \\
Peptide library 1 & EPIYMFFF & $1^{\text {st }}$ preference \\
& VV IIII & $2^{\text {nd }}$ preference \\
& L F L & $3^{\text {rd }}$ preference \\
\hline
\end{tabular}

Table 1 CSK phosphorylation sites

The tyrosine phosphorylation site is underlined and labeled in red. The acidic residues in c-Src interacting with the arginine residues in $\alpha$ helix D and $\alpha \mathrm{F}-\alpha \mathrm{G}$ loop of CSK are underlined. 


\begin{tabular}{|c|c|c|}
\hline \multicolumn{2}{|c|}{ Phosphorylation site } & Sequence \\
\hline \multirow{5}{*}{ SHPS-1 } & c-Src(Tyr527) & EDYFTSTEPQYYQPGNL \\
\hline & Tyr-428 & DTNDITYADLNLPK \\
\hline & Tyr-452 & PNNHTEYASIQTSP \\
\hline & Tyr-469 & SEDTLTY YADLDMVH \\
\hline & Tyr-495 & EPSFSEYASVQV \\
\hline
\end{tabular}

Table 2 CHK phosphorylation sites 
A.

fatty acid

acylation motif $Y_{\mathrm{u}}$

spe Unique

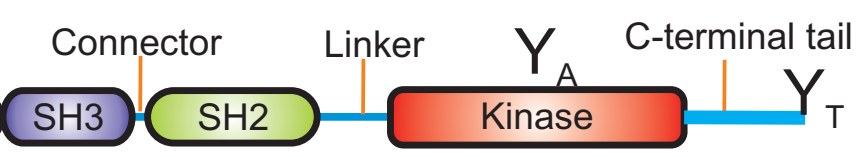

B.

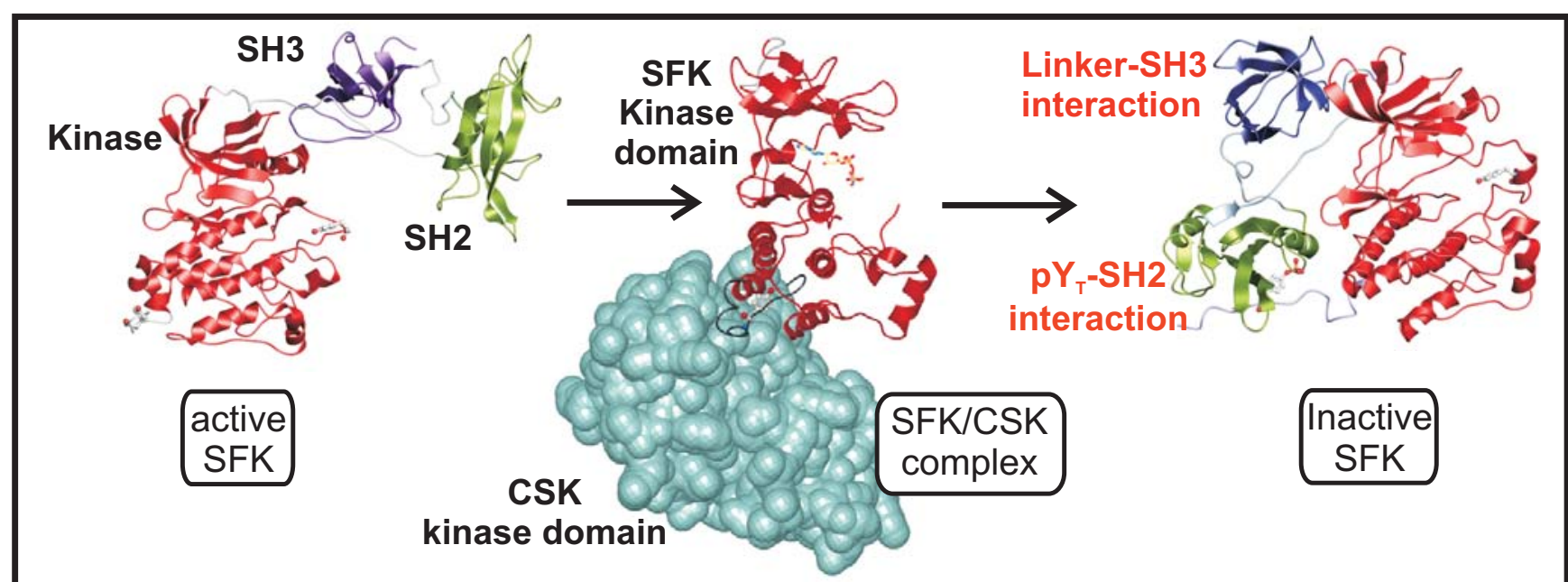

C.

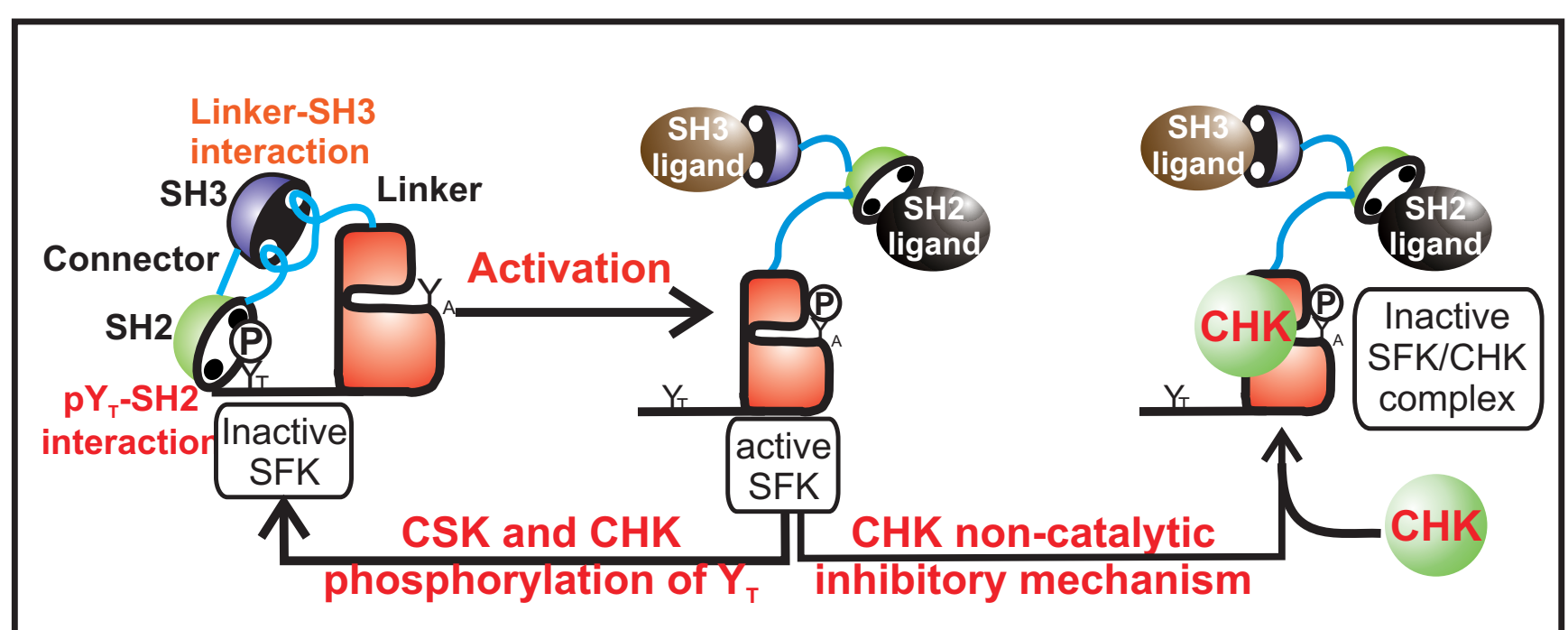

Fig. 1 
A.

\section{$\mathrm{SH} 3-\mathrm{SH} 2$}

SH2-kinase

Connector Linker

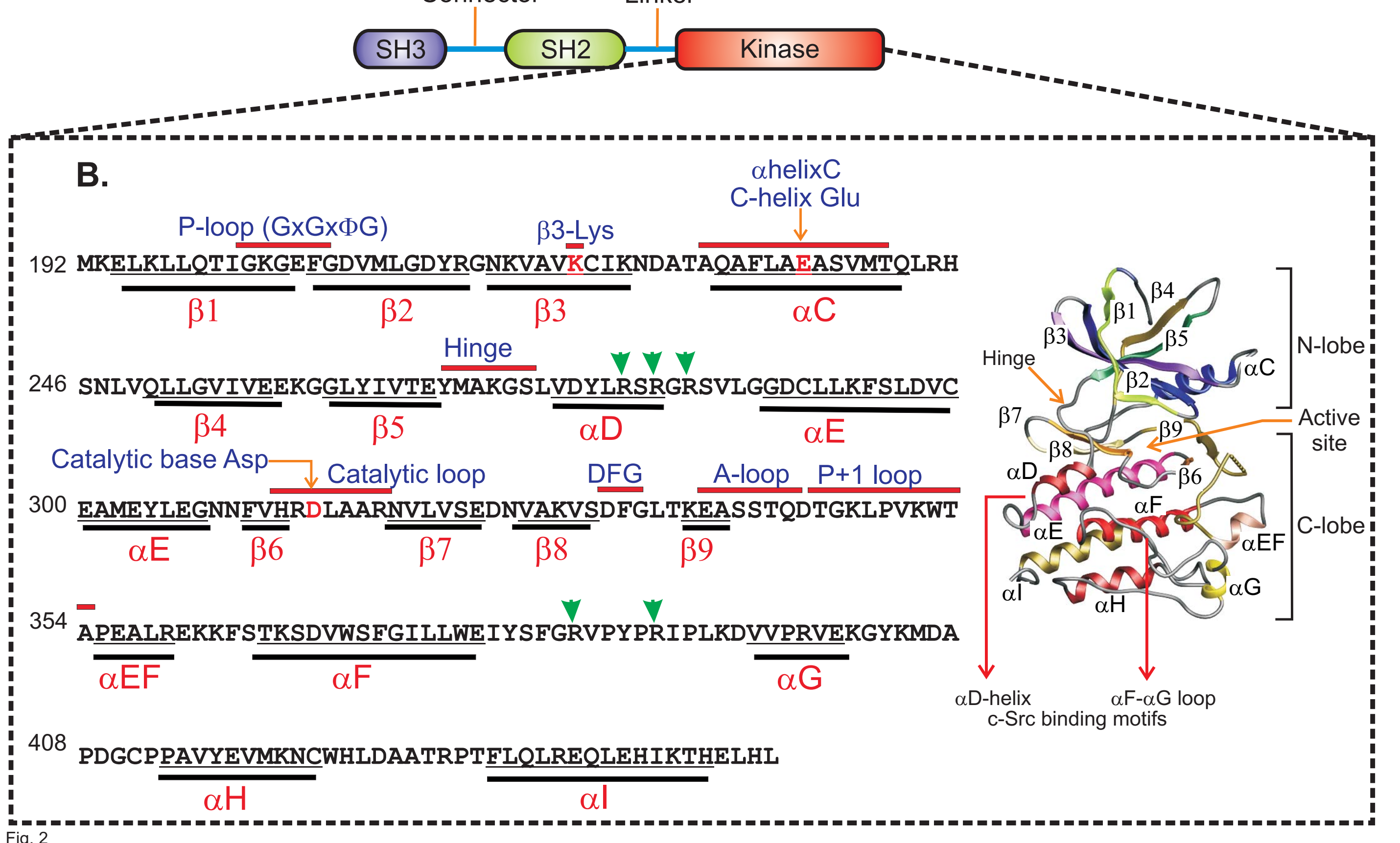


Active

A.

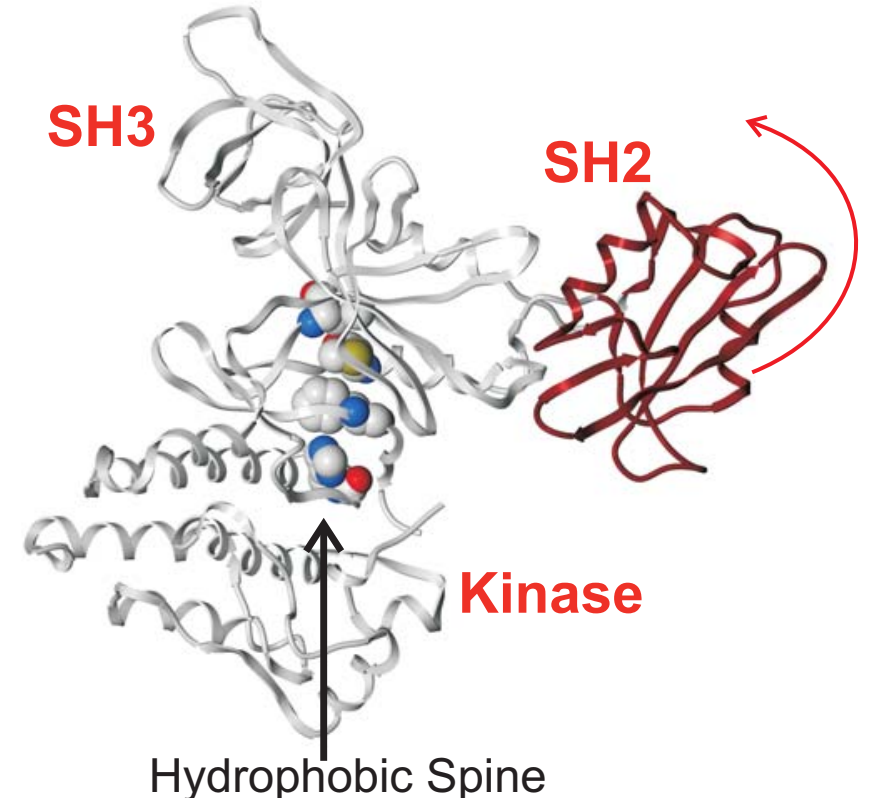

C.

Hydrophobic

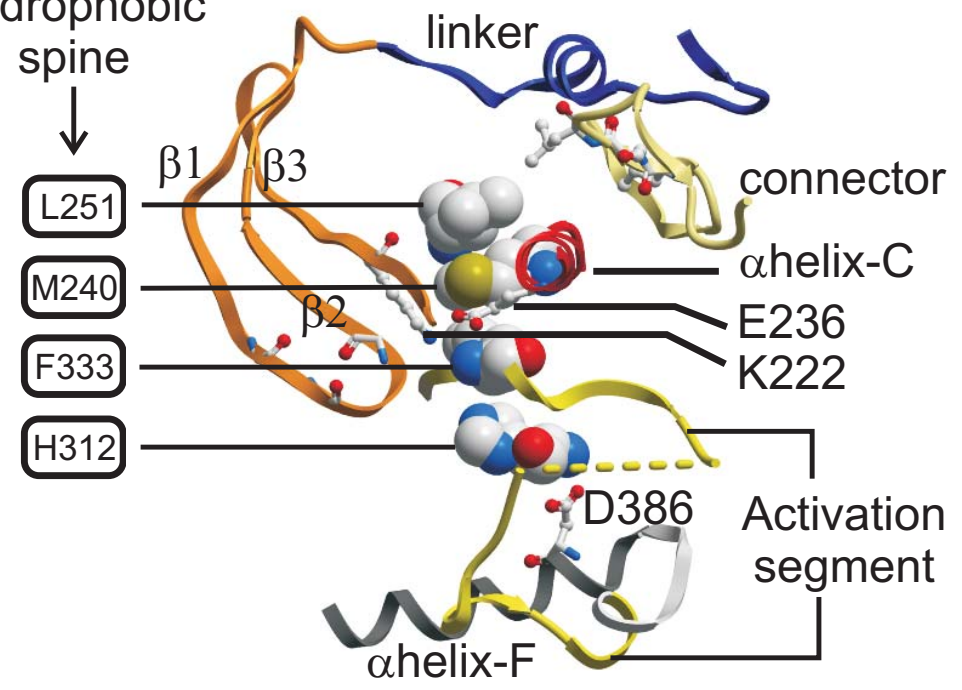

Inactive

B.
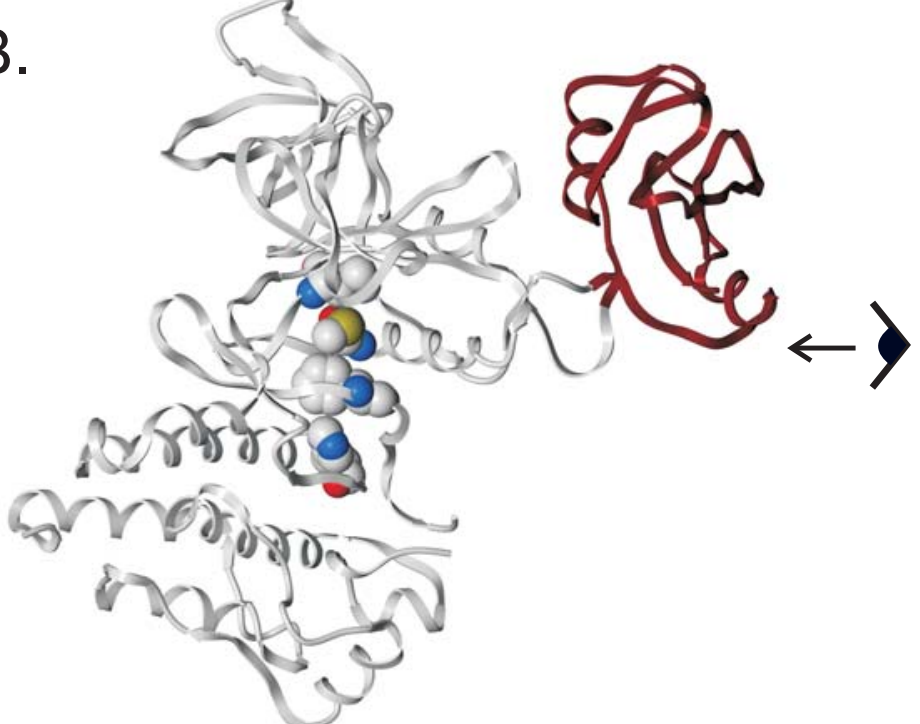

D.

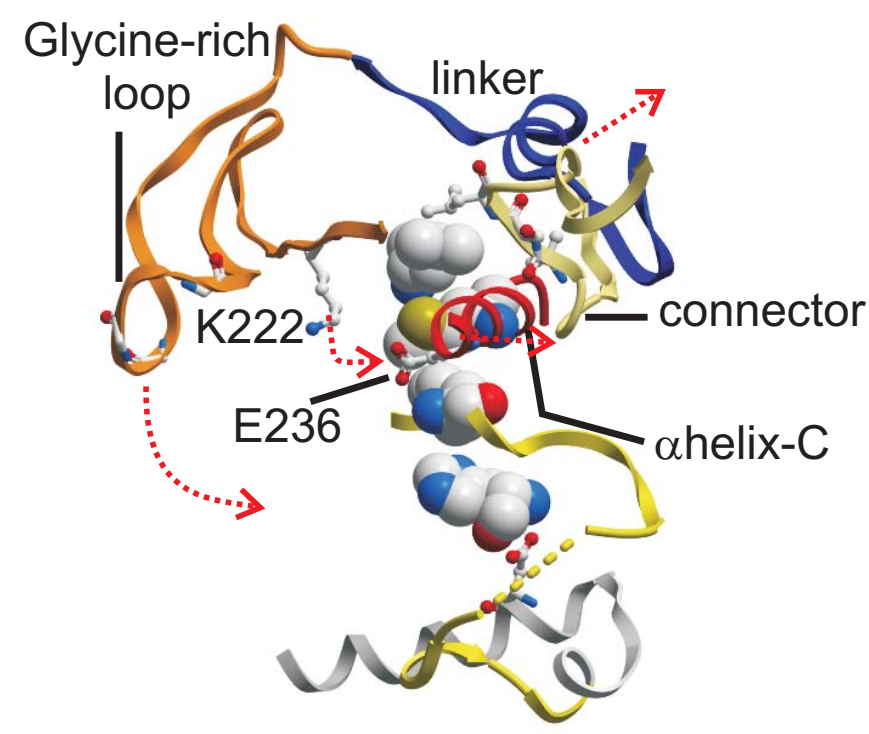




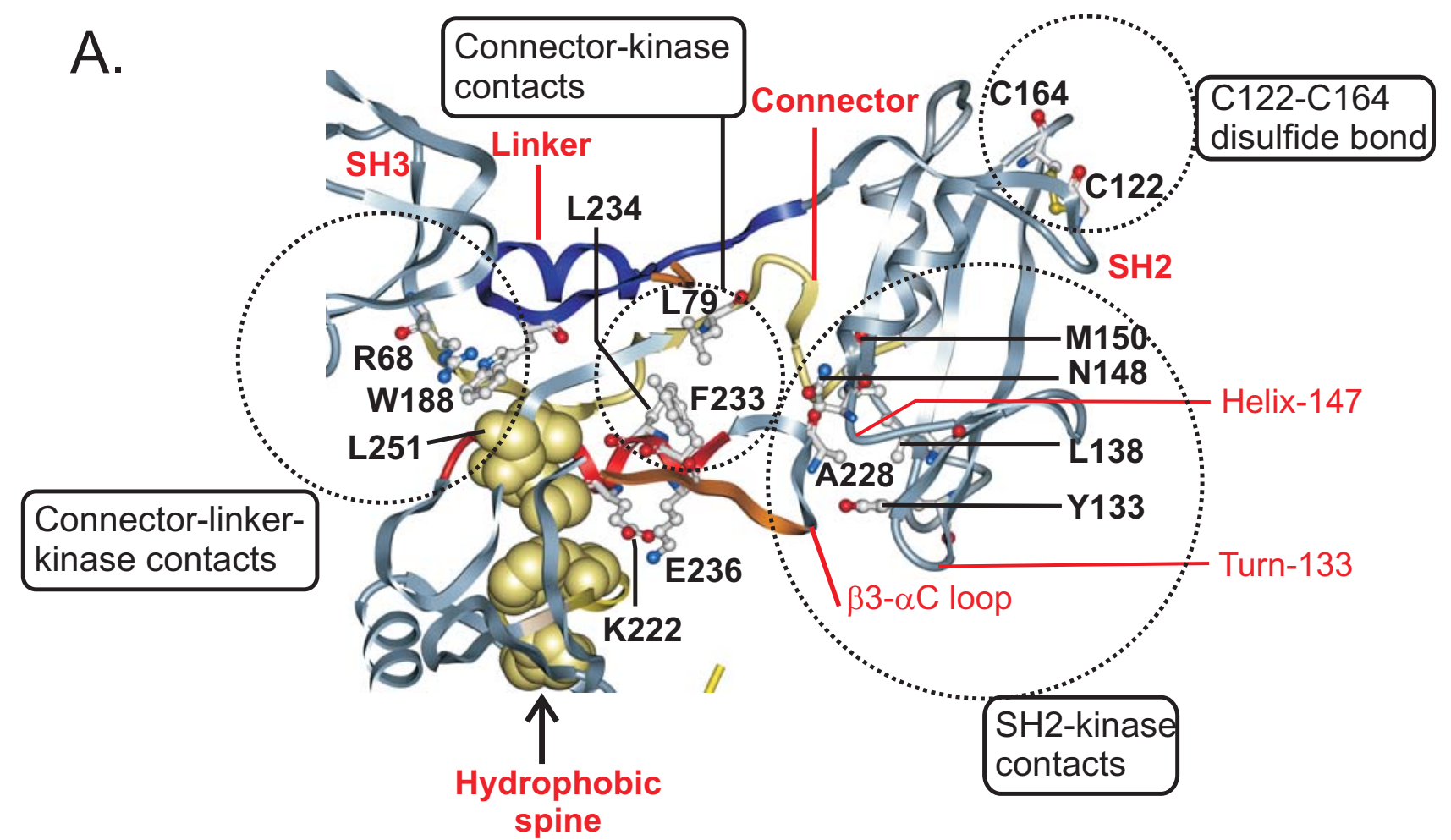

B.

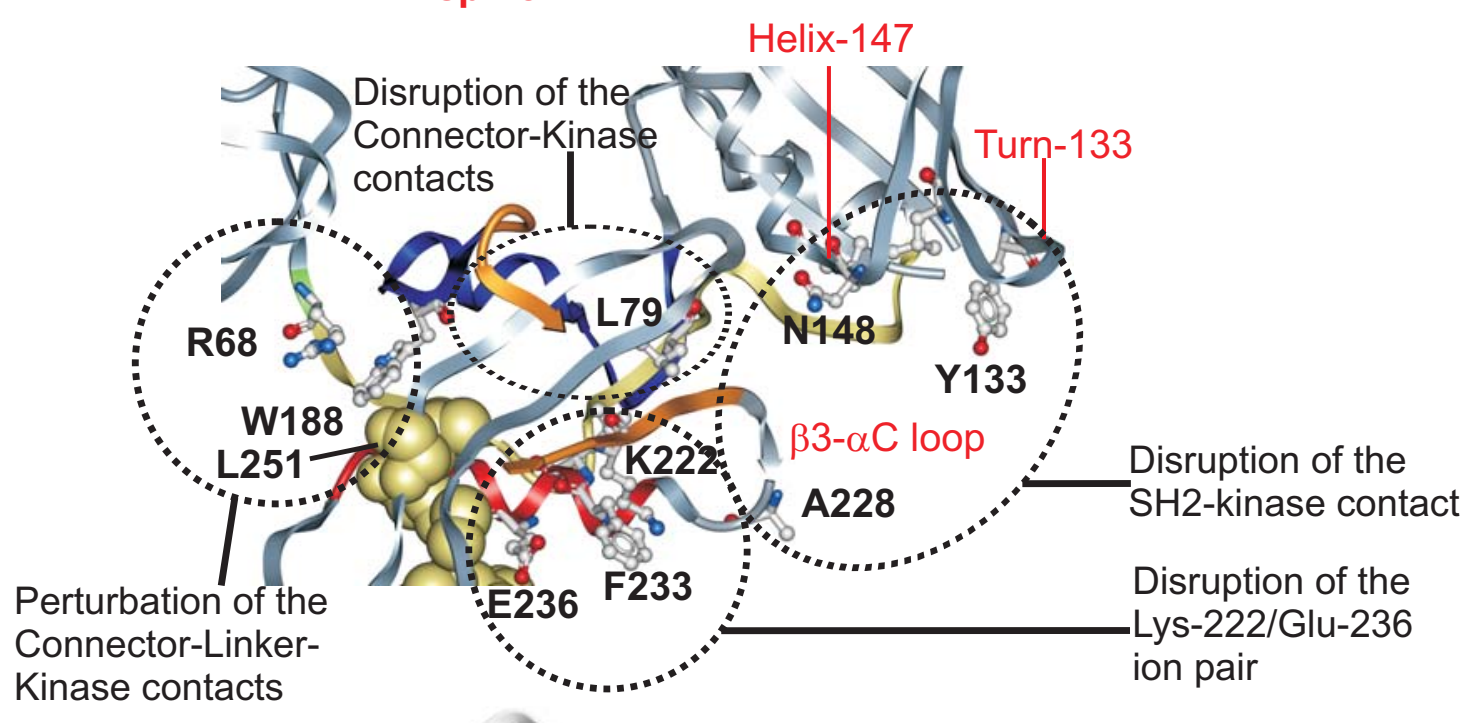

C.

Fig. 4

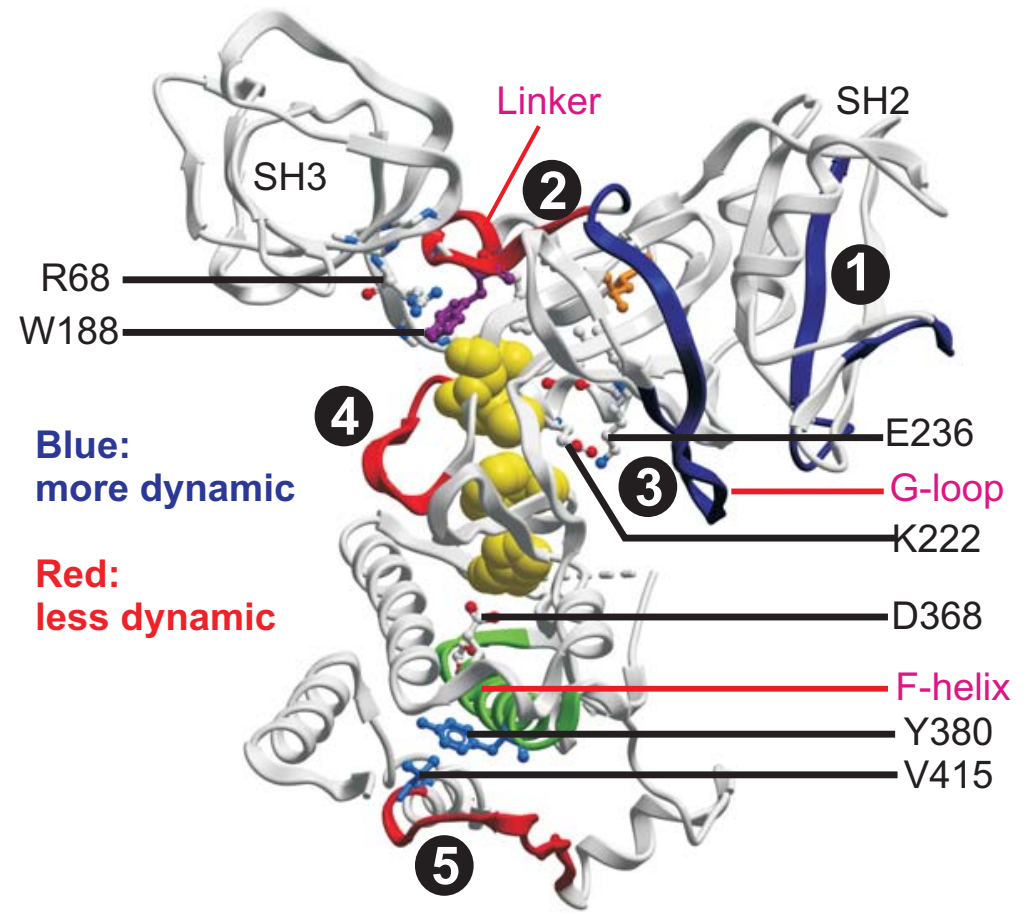


A.
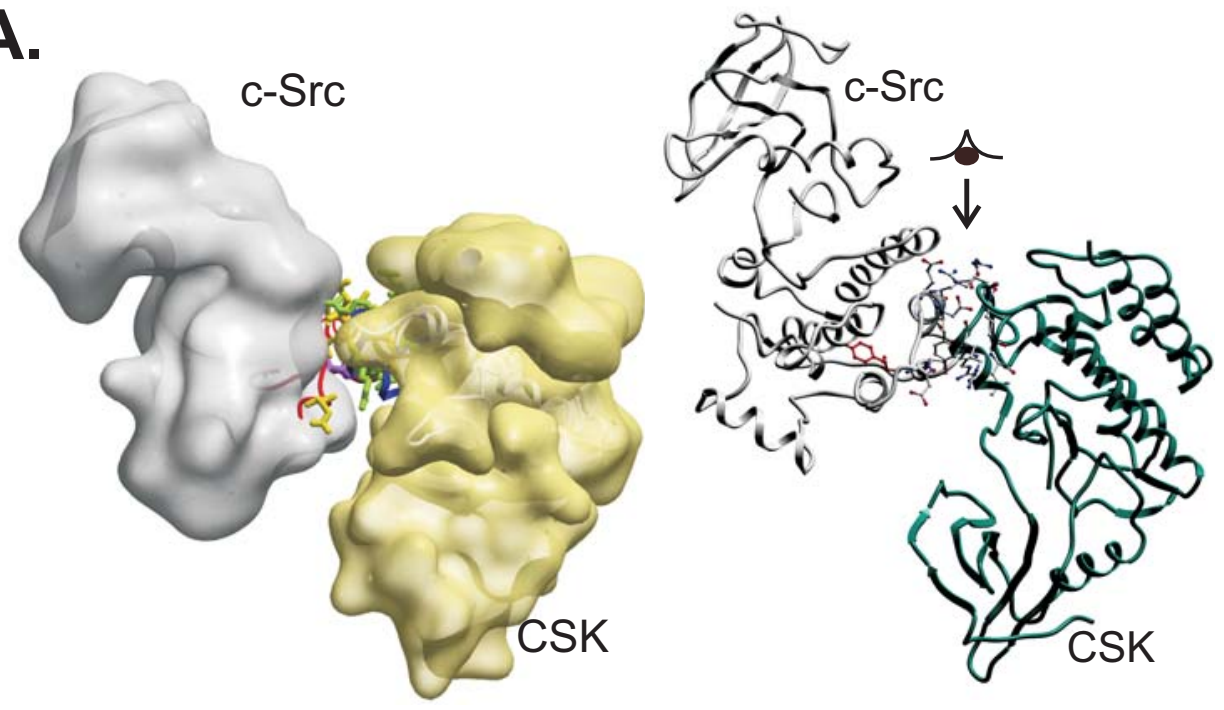

C.

$$
\stackrel{\alpha \text { l helix }}{\longrightarrow} \underset{\text { C-terminal tail }}{\longrightarrow} \longrightarrow
$$

C-SrC ${ }^{504}$ EERPTFEYLQAFLEDYFTSTEPQYQPGENL ${ }^{53}$

Lyn ${ }^{415}$ EERPTFDYLQSVLDDFYTATEGQYQQQP ${ }^{512}$

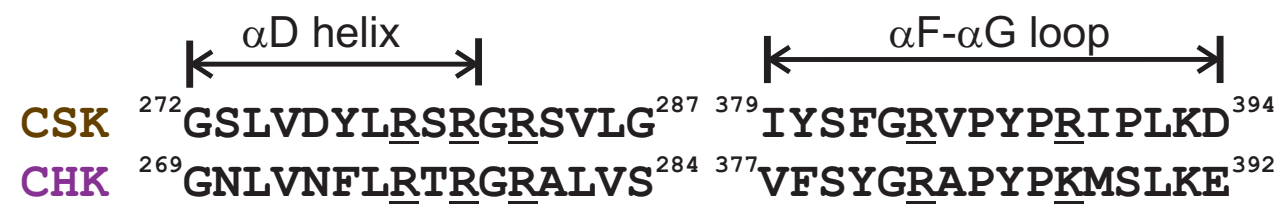

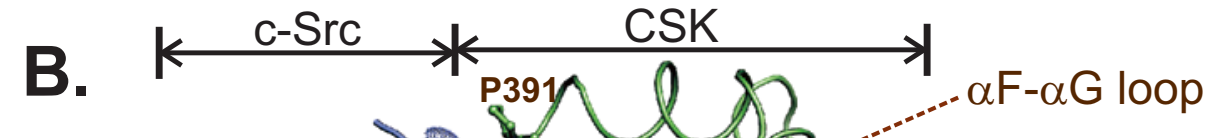

al helix

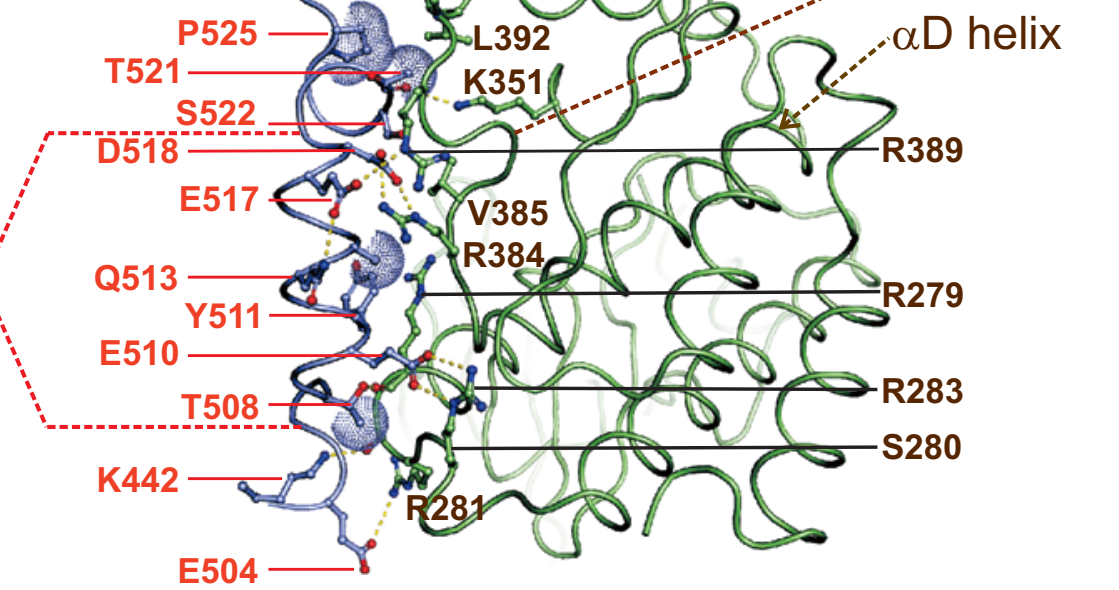

D.

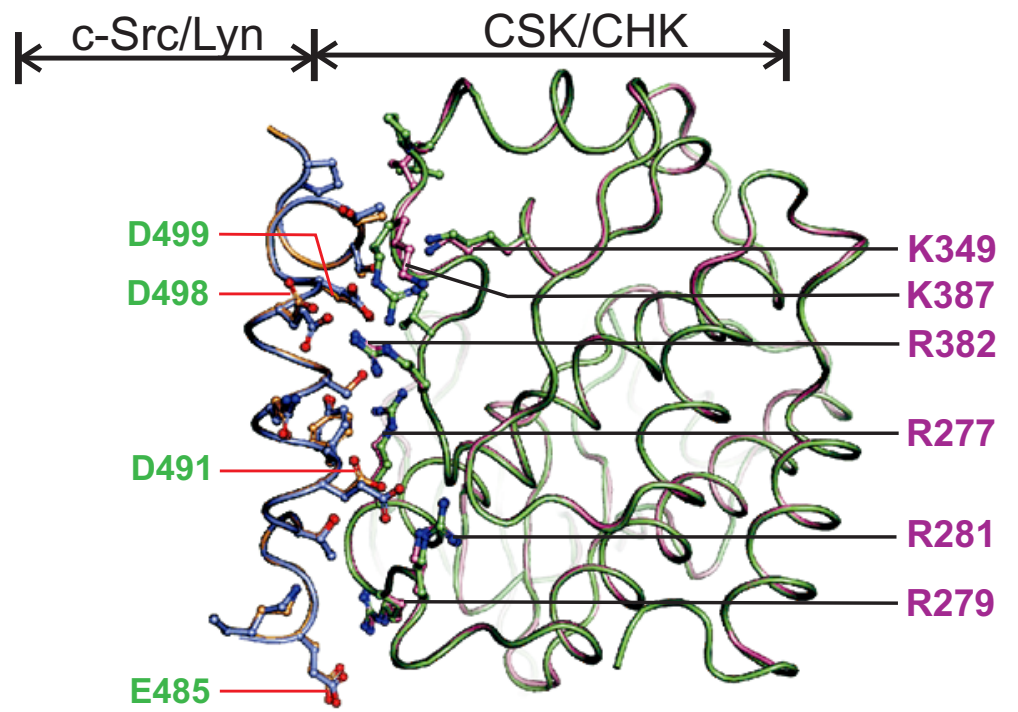




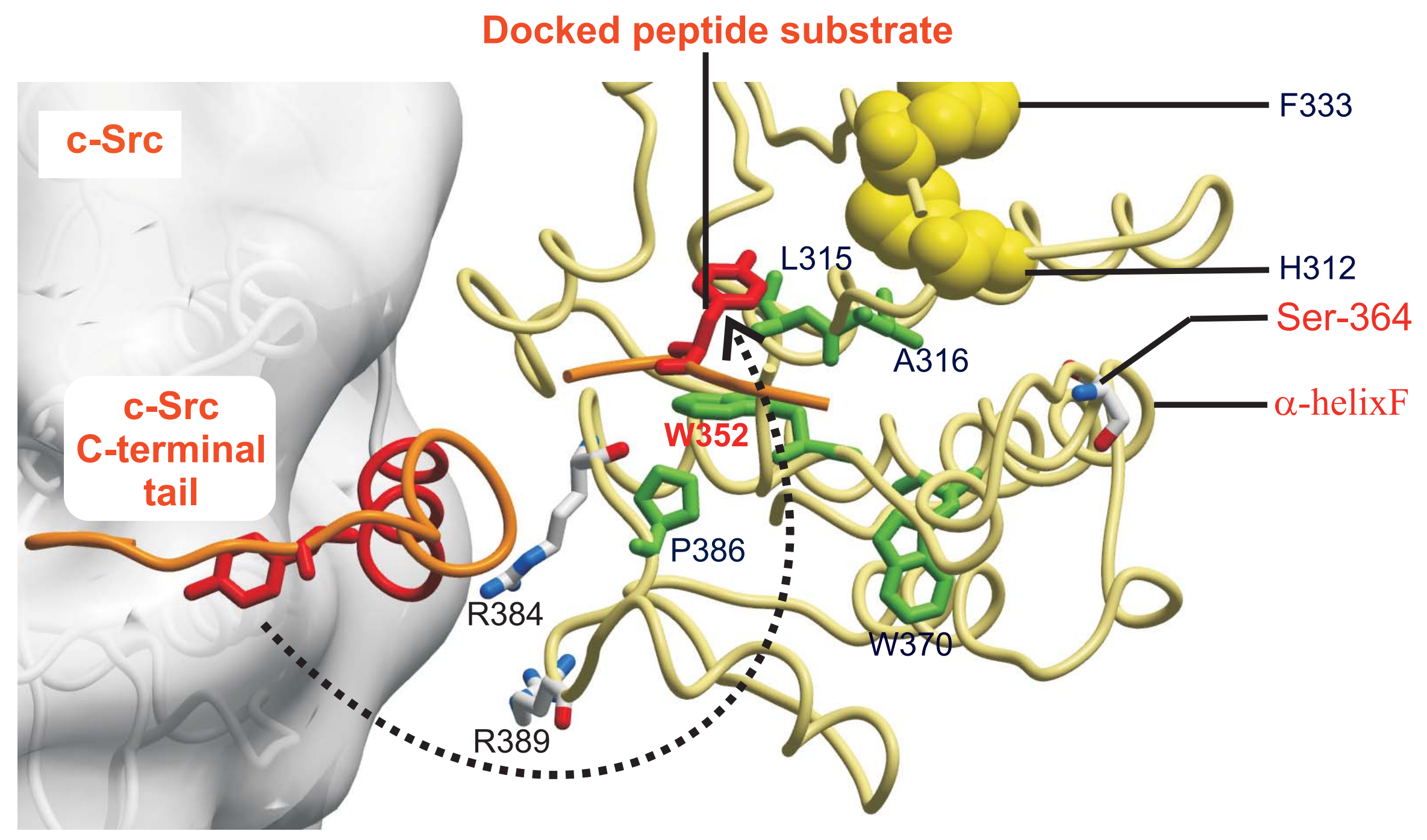

Fig. 6 
A.

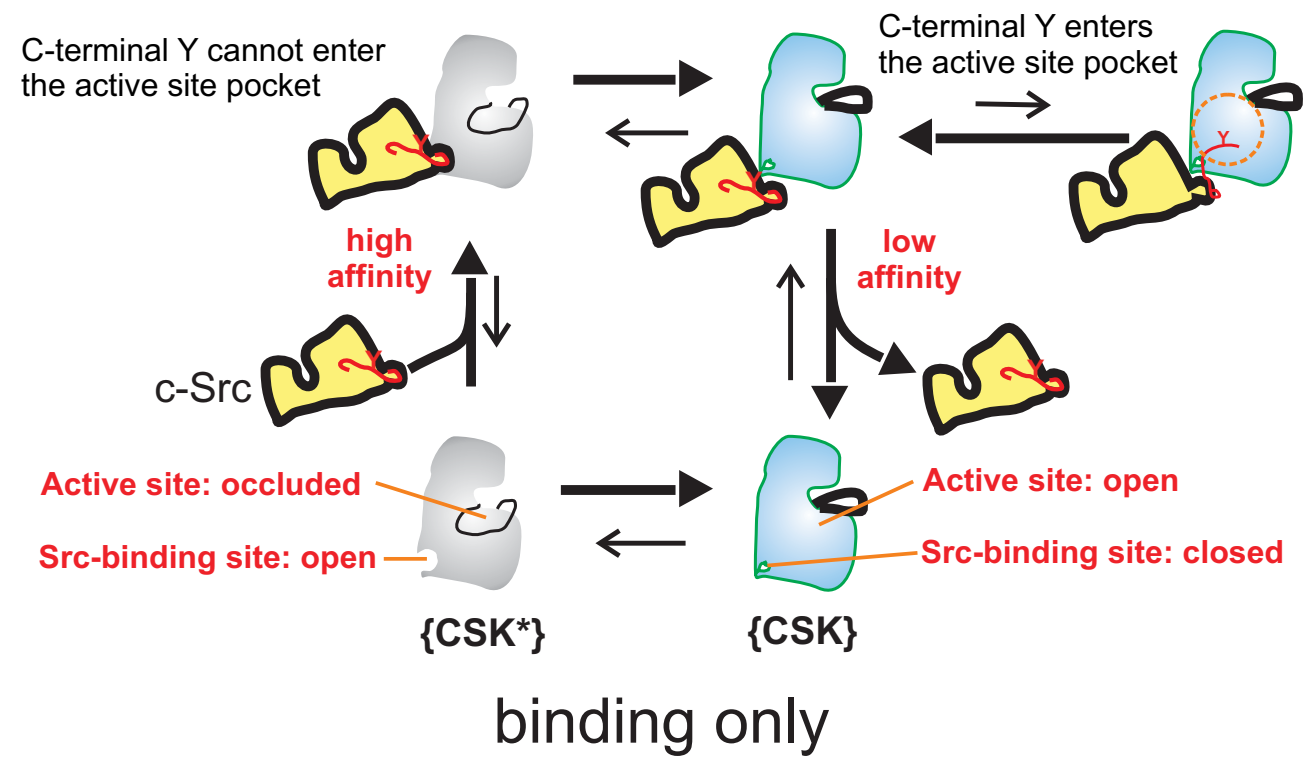

B.

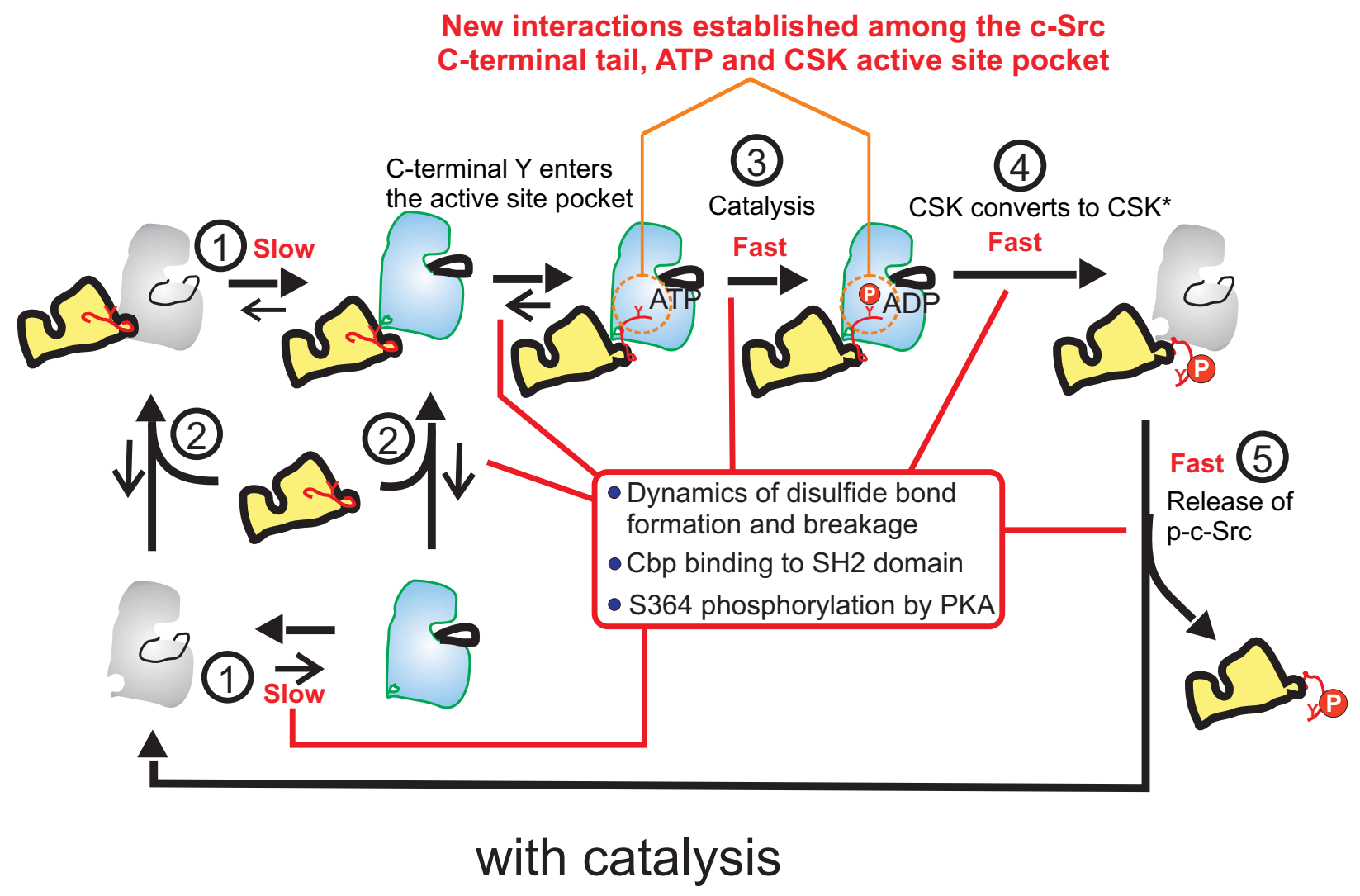

Fig. 7 


\section{Supplemental Data}

Structural Elements and Allosteric Mechanism Governing Regulation and Catalysis of CSK-Family Kinases and Their Inhibition of Src-Family Kinases

K. K. Ia et al.

SFig. $1 \quad$ Alignment of CSK and CHK sequences

SFig. 2 A model of the CSK dimer

SFig. 3 Alignment of protein kinase domain sequences of CSK, CHK, c-Src, IGF1receptor kinase, insulin receptor kinase and PKA

SFig. 4 The "hydrophobic cradle" formed by the P+1 loop of the activation segment and $\alpha \mathrm{F}-\alpha \mathrm{G}$ loop of CSK for interactions with hydrophobic residues in the peptide substrate

SFig. 5 Sequence alignment of Src-family kinases, Frk-family kinases and representatives of other tyrosine kinase families for the region corresponding to c-Src residues 499-533 
CSK

CHK

CSK

CHK

CSK

CHK

CSK

CHK

CSK

CHK

CSK

CHK

CSK

CHK

CSK

CHK
SH3 domain

MSAIQASW[PSGTECIAKYNFHGTAEQDLPFCKGDVLTIVAVTKDPNWYKAKNK-VGREGI 59

--MPTQRW[APGTQCMTKCENSRPKPGELAFRKGDMVTILEACEDKSWYRAKHHSSGQEGL 58

$\mathrm{SH} 3-\mathrm{SH} 2$ connector

SH2 domain

IPANYVQK]REGVKAGTKLSLMP[WFHGIITREQAERLLYPPETGLFLVRESTNYPGDYTLC 119

LAAAALRQ]REALSTDPKLSLMP[WFHGKISGQEAIQQLQPPEDGLFLVRESARHPGDYVLC 118

VSCEGKVEHYRIMYHASKLSIDEEVYFENLMQLVEHYTTDADGLCTRLIKP]KVMEGTVAA 179 VSFGRDVIHYRVLHRDGHLTIDEAVCFCNLMDMVEHYTRDKGAICTKLVKP]KRKQGAKSA 178

SH2-kinase Tinker

QDEFYRSGWALNMKE[LKLLQTIGKGEFGDVMLGDYRGNKVAVKCIKNDATAQAFLAEASV 239

EEELAKAGWLLDLQH[LTLGAQIGEGEFGAVLQGEYLGQKVAVKNIKCDVTAQAFLDETAV 238

Kinase domain

MTQLRHSNLVQLLGVIVEEKGGLYIVTEYMAKGSLVDYLRSRGRSVLGGDCLLKFSLDVC 299

MTKLQHRNLVRLLGVILHH--GLYIVMEHVSKGNLVNFLRTRGRALVSTSQLLQFALHVA 296

EAMEYLEGNNFVHRDLAARNVLVSEDNVAKVSDFGLTKEASST-QDTGKLPVKWTAPEAL 358 EGMEYLESKKLVHRDLAARNILVSEDLVAKVSDFGLAKAELRKGLDSSRLPVKWTAPEAL 356

REKKFSTKSDVWSFGILLWEIYSFGRVPYPRIPLKDVVPRVEKGYKMDAPDGCPPAVYDV KNGRFSSKSDVWSFGVLLWEVFSYGRAPYPKMSLKEVSEAVEKGYRMEPPDSCPGPVHTL

SFig. 1 Alignment of CSK and CHK sequences - The SH3, SH2 and kinase domains as well as the SH3-SH2 connector and SH2-kinase linker are colour-coded as shown in SFig. 3. The boundaries of each functional domain is marked by the brackets ([ and ]), For the CSK SH3 domain, the conserved aromatic residues including Y18, F20, W47 and Y64 essential for binding the PxxP motif are underlined. For CSK and CHK kinase domains, the five conserved arginine and lysine residues in the $\alpha \mathrm{D}$-helix and $\alpha \mathrm{F}-\alpha \mathrm{G}$ loop essential for binding of c-Src are underlined. 

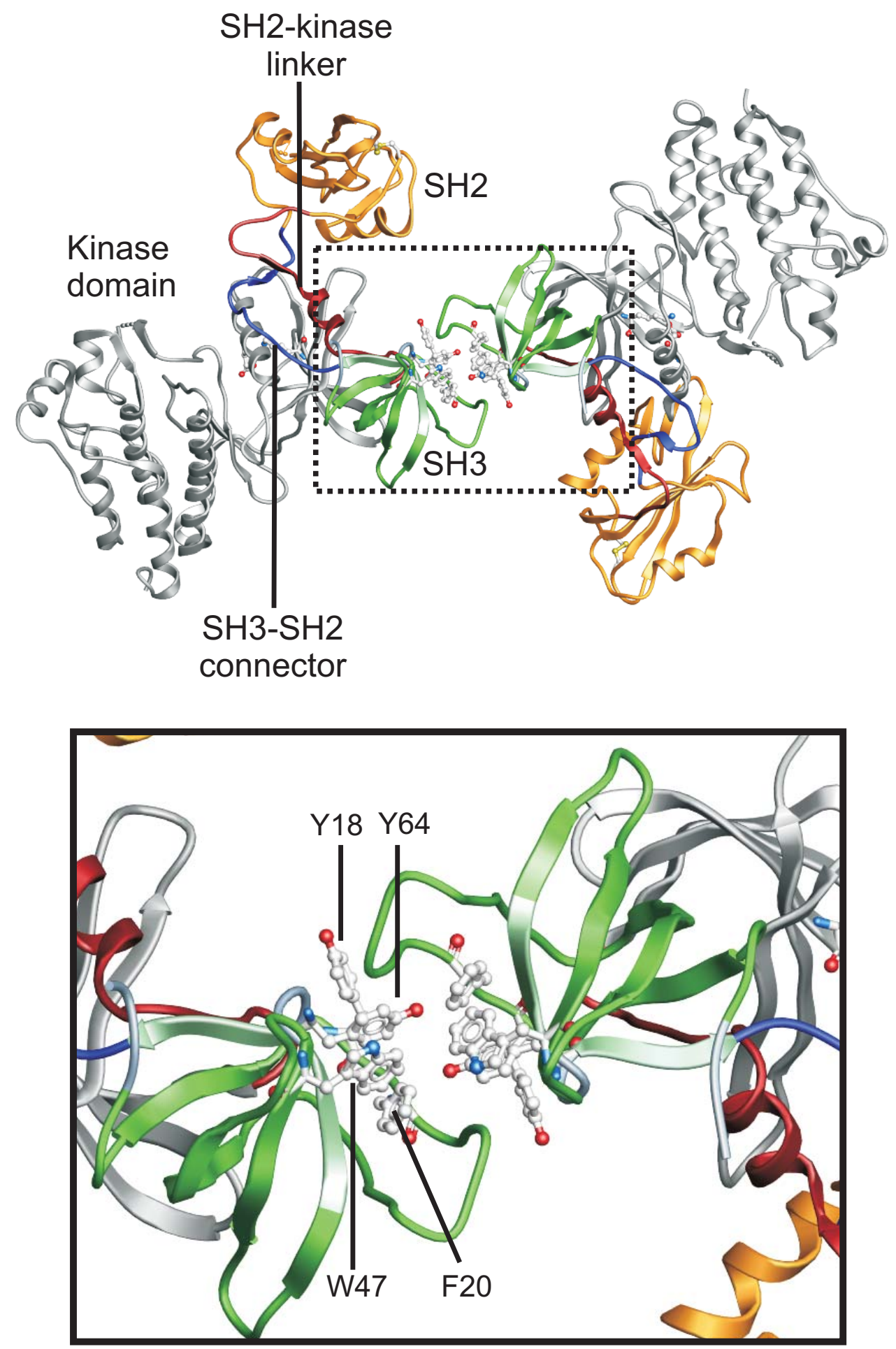

\section{Sfig. 2 A Model of the CSK Dimer}

A. A pair of CSK monomers (Structures $A$ and $D$ of pdb entry $1 \mathrm{~K} 9 \mathrm{~A}$ ) dimerise by interactions of their SH3 domains. B. The close-up view of the boxed area in panel A. The four conserved aromatic hydrophobic residues involved in binding the PxxP motif of SH3 ligands are shown (refer to SFig. 1 for their identities). These hydrophobic residues mediate the interactions between the $\mathrm{SH} 3$ domains in the CSK dimer. 


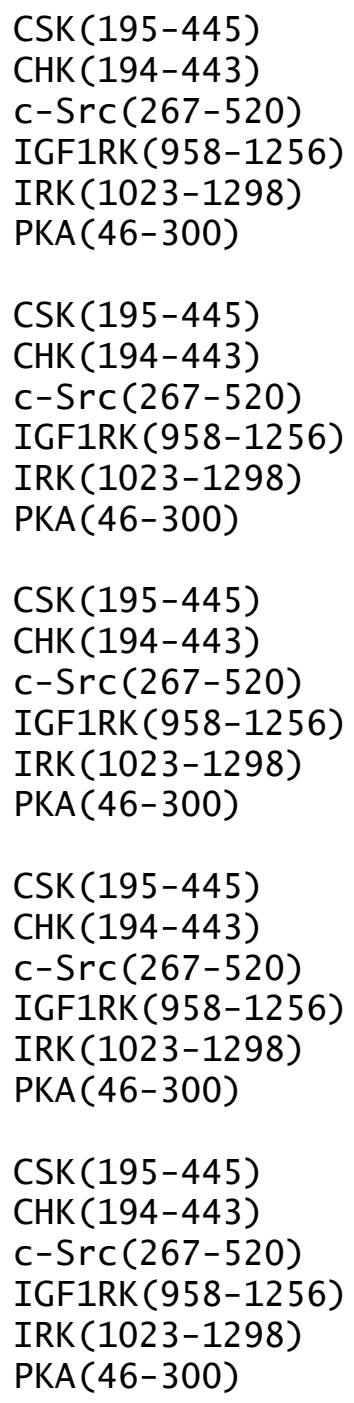

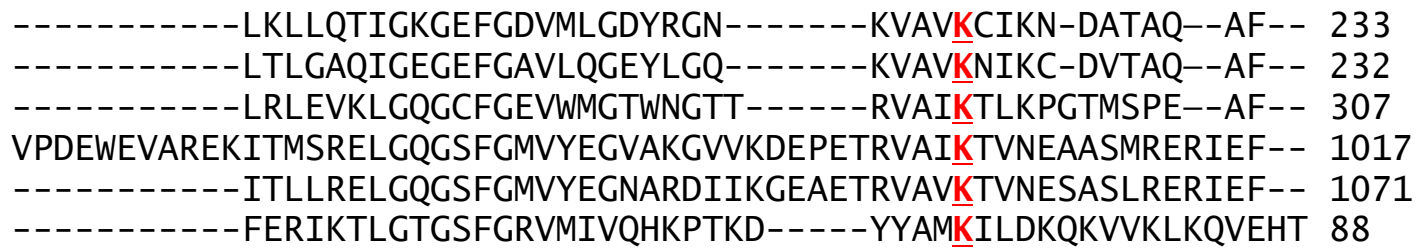

LAEASVMTQLRHSNLVQLLGVIVEEKGGLYIVTEYMAKGSLVDYLRSR--------GRSV 285 LDETAVMTKLQHRNLVRLLGVILHH--GLYIVMEHVSKGNLVNFLRTR-------GRAL 282

LQEAQVMKKLRHEKLVQLYAVVSEE--PIYIVTEYMSKCSLLDFLKGE-------MGKY 357 LNEASVMKEFNCHHVVRLLGVVSQGQ-PTLVIMELMTRGDLKSYLRSLRPEMENNPVLAP 1076 LNEASVMKGFTCHHVVRLLGVVSKGQ-PTLVVMELMAHGDLKSYLRSLRPEAENNPGRPP 1130 LNEKRILQAIQFPFLVSLRYHFKDNS-NLYMVLEYVPGGEMFSHLRKVG---------R 137

LGGDCLLKFSLDVCEAMEYLEGNNFVHRDLAARNVLVSEDNVAKVSDFGLT----KEASS 341 VSTSQLLQFALHVAEGMEYLESKKLVHRDLAARNILVSEDLVAKVSDFGLA----KAELR 338 LRLPQLVDMAAQIASGMAYVERMNYVHRDLRAANILVGENLVCKVADFGLAR-LIEDNEY 416 PSLSKMIQMAGEIADGMAYLNANKFVHRDLAARNCMVAEDFTVKIGDFGMTRDIYETDYY 1136 PTLQEMIQMAAEIADGMAYLNAKKFVHRDLAARNCMVAHDFTVKIGDFGMTRDIYETDYY 1190 FSEPHSRFYAAQIVLAFEYLHYLDLIYRDLKPENLLIDSQGYLKVTDFGFAKRVKGRTWT 197

T-QDTCKLPVKWTAPEALREKKFSTKSDVWSFGILLWEIYSFGRVPYPRIPLKDVVPRVE 400 KGLDSSRLPVKWTAPEALKNGRFSSKSDVWSFGVLLWEVFSYGRAPYPKMSLKEVSEAVE 398 TARQGAKFPIKWTAPEAALYGRFTIKSDVWSFGILLTELTTKGRVPYPGMVNREVLDQVE 476 RKGGKGLLPVRWMSPESLKDGVFTTYSDVWSFGVVLWEIATLAEQPYQGLSNEQVLRFVM 1196 RKGGKGLLPVRWMAPESLKDGVFTTSSDMWSFGVVLWEITSLAEQPYQGLSNEQVLKFVM 1250 LCG-----TPEYLAPEIILSKGYNKAVDWWALGVLVYEMAAG-YPPFFADQPIQIYEKIV 251

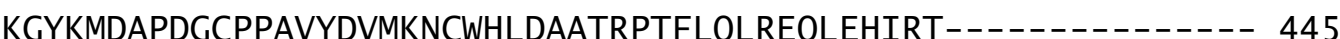

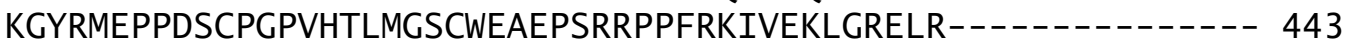
RGYRMPCPPECPESLHDLMCQCWRKDPEERPTFEYLQAFLEDYF--.-EGGLLDKPDNCPDMLLELMRMCWQYNPKMRPSFLEIISSIKEEMEPGFREVSFYYSEENK 1256 DGGYLDQPDNCPERVTDLMRMCWQFNPKMRPTFLEIVNLLKDDLHPSF--------- 1298 SG-KVRFPSHFGSDLKDLLRNLLQVDLTKR--YGNLKAGVNDIKNQKWF---------- 297

SFig. 3 Alignment of protein kinase domains of CSK, CHK, c-Src, IGF1-receptor kinase, insulin receptor kinase and PKA - The boundaries of ths kinase domains are indicated by residue numbers in the parentheses. All essential residues for catalysis are highlighted in red fonts. The activation segment that begins with the DFG motif and ends with the APE motif is indicated. The c-Src binding motifs in the $\alpha \mathrm{D}$ helix and $\alpha \mathrm{F}-\alpha \mathrm{G}$ loop are underlined and the essential arginines labeled with green fonts. 
SFig. 4 The "hydrophobic cradle" formed by the P+1 loop of the activation segment and $\alpha \mathrm{F}-\alpha \mathrm{G}$ loop of CSK for interactions with hydrophobic residues in the peptide substrate

A. The structure of active conformation of CSK (PDB entry: 1K9A) with the peptide substrate of the IGF1RK (PDB entry: 1K3A) docked to the active site. The docking involves superimposing the C-terminal lobes of both the IGF1RK and CSK by the "magic fit" function of spdviewer.

B. A birds' eye view of the structure shown in panel A. The activation segment is coloured in orange. The ribbon showing the backbone of the docked peptide substrate is coloured in green.

C. The close-up view of the area marked by the box in panel $\mathrm{B}$. The amino acid residues in the docked peptide are labeled as $\mathrm{P}$ for the phosphorylation site tyrosine, $\mathrm{P}-1, \mathrm{P}-2, \mathrm{P}-3$ and $\mathrm{P}-4$ for the residues located at the $\mathrm{N}$-terminal side of the $\mathrm{P}$ site, and $\mathrm{P}+1, \mathrm{P}+2$ and $\mathrm{P}+3$ for residues located at the $\mathrm{C}$-terminal side of the $\mathrm{P}$ site.

D. The optimal CSK phosphorylation sequence and the residues in the revealed by Panel C that are involved in forming the "hydrophobic cradle" for binding to the hydrophobic residues in the $\mathrm{P}-1, \mathrm{P}+1, \mathrm{P}+2$ and $\mathrm{P}+3$ positions of the optimal CSK phosphorylation sequence. 
A.

B.
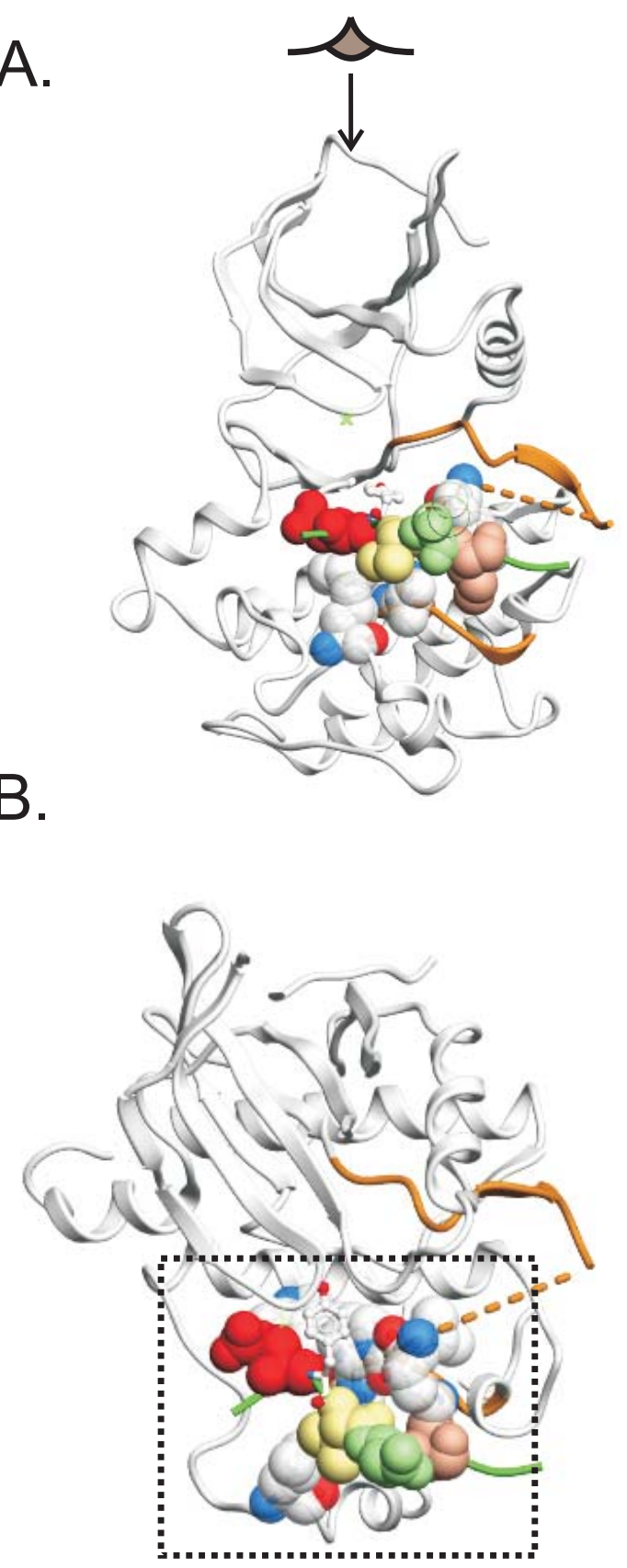

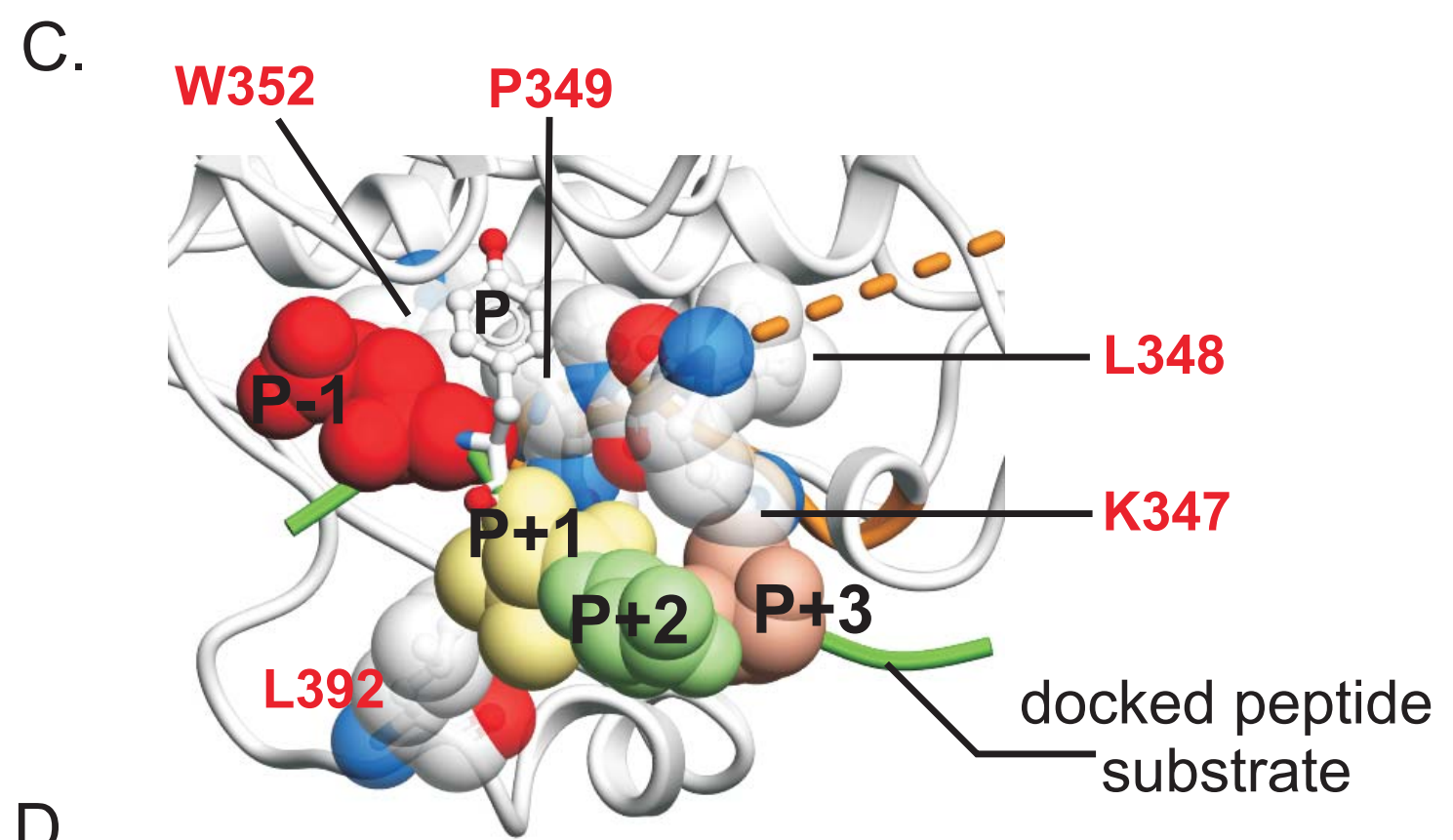

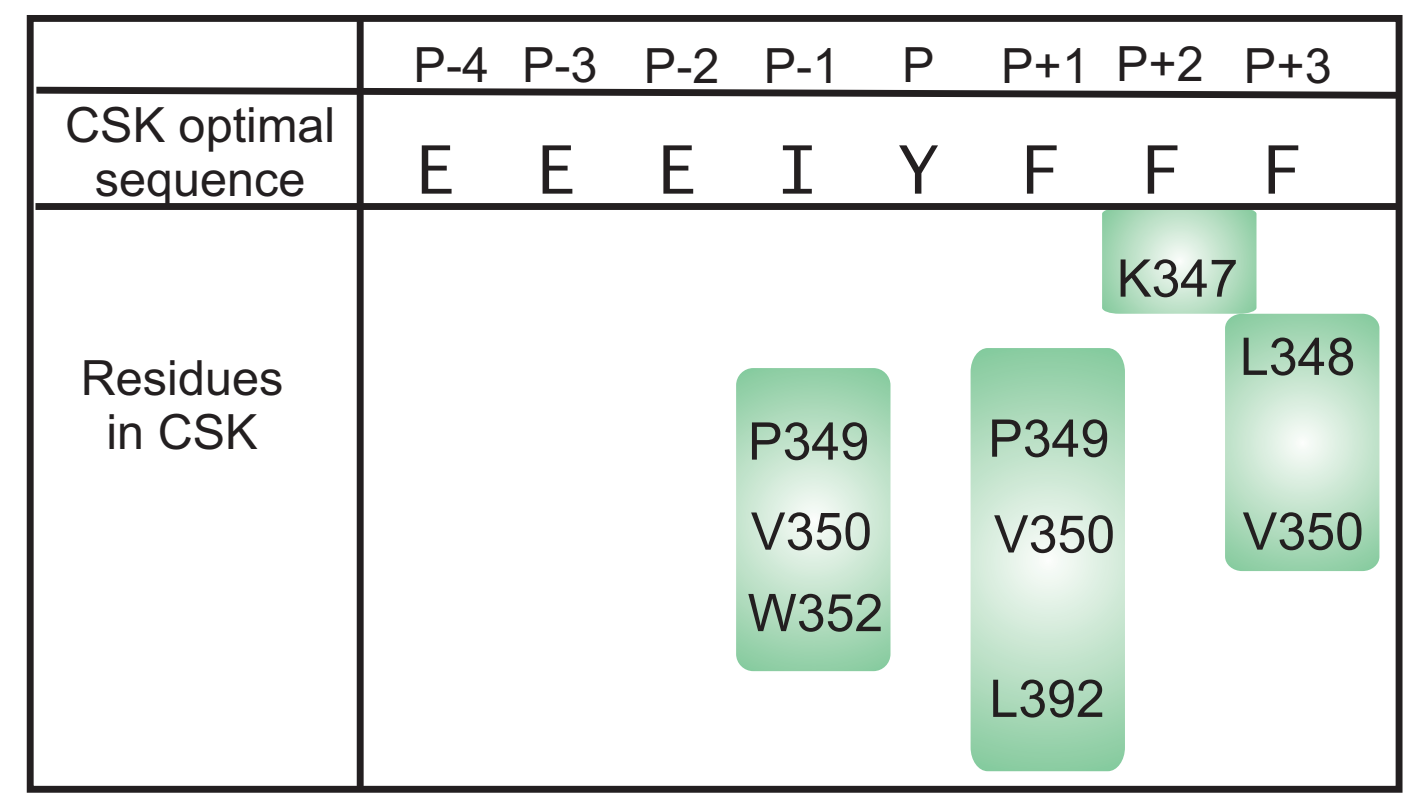

SFig.4 


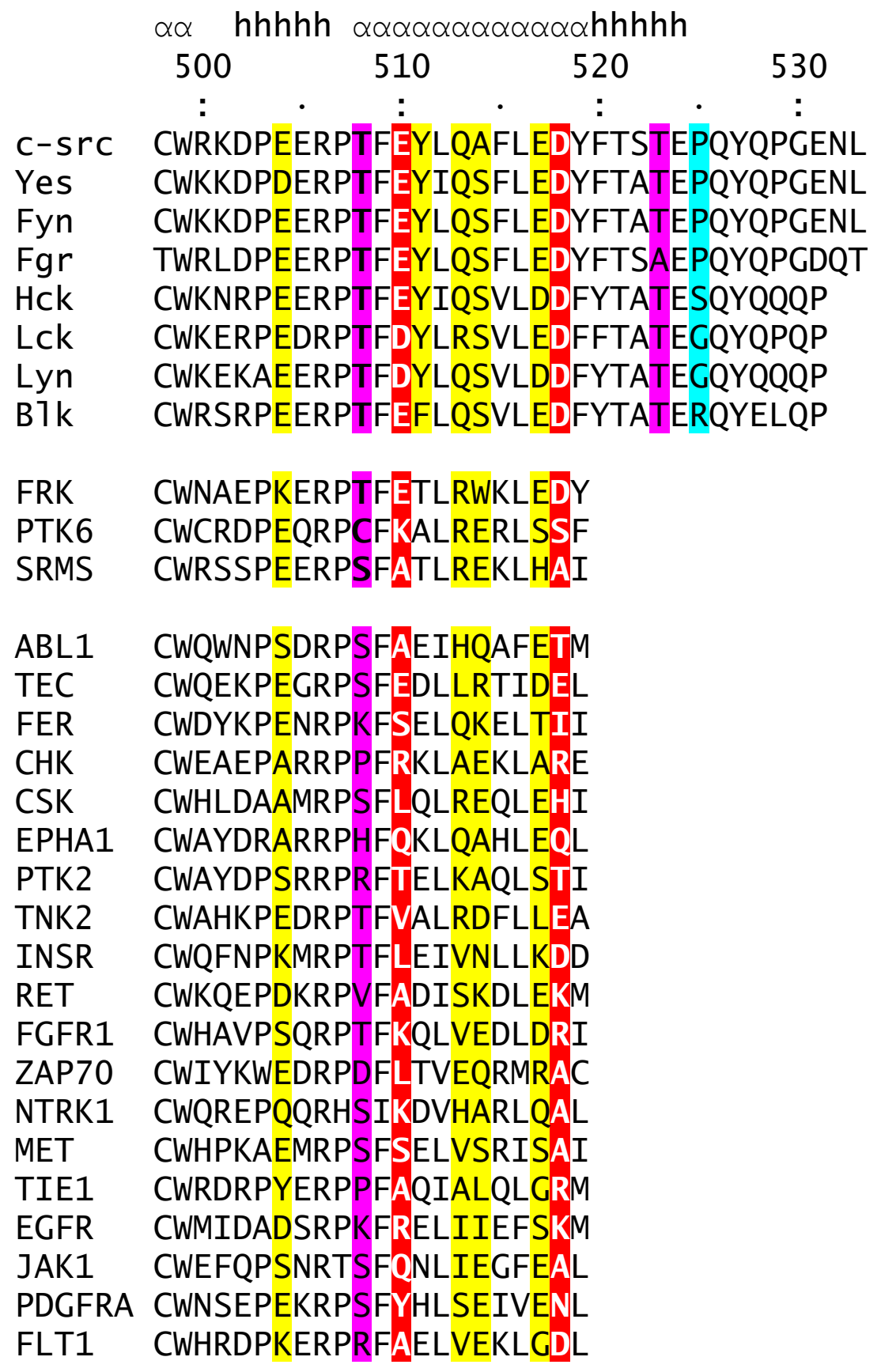

SFig. 5 Sequence alignment of Src family kinases, Frk-family kinases and representatives of other tyrosine kinase families for the region corresponding to cSrc residues 499-533. 